\title{
Evolution métamorphique des métapélites du Massif hercynien des Rehamna (Maroc): implications tectonothermales
}

\author{
ABDEL MOUHSINE AGHZER' et RICARDO ARENAS ${ }^{2}$ \\ 'Département de Géologie, Faculté des Sciences, Université Chouaib Doukkali, BP 20, \\ 24000 Eljadida, Maroc \\ ${ }^{2}$ Departamento de Petrología y Geoquímica, Facultad de Geología, \\ Universidad Complutense, 28040 Madrid, Espagne
}

\begin{abstract}
Résumé-L'épaississement crustal maximal des Rehamna centraux est acqui par le chevauchement d'Ouled Zedness, qui superpose la formation de Jorf El Ahmar (Rehamna orientaux) à la nappe des Skhour (Rehamna centraux) sous un climat métamorphique de moyenne $P / T$ culminant dans le faciès amphibolite. Une faille normale ductile, en l'occurrence la faille d'El Broumi, provoque ensuite la mise en place, sous des conditions métamorphiques rétrogrades, d'un bloc dévono-viséen (formations d'El Broumi-Ouled Hassine et de Lalla Tittaf), métamorphisé dans le faciès amphibolite supérieur, au sommet de l'édifice structural. L'étude pétro-structurale des assemblages minéralogiques syn-pic thermique indique que la formation des Skhour et les formations dévono-viséennes ont subi des évolutions tectonométamorphiques apparement similaires. Les caractéristiques chimiques des phases des assemblages syn-pic thermique plaident en faveur d'un métamorphisme plus intense dans la première formation. La quantification des conditions $P-T$ du pic thermique régional $\left(560^{\circ} \mathrm{C}\right.$ à $9 \mathrm{kbar}$ dans la nappe des Skhour, et $532^{\circ} \mathrm{C}$ à $7 \mathrm{kbar}$ dans les Rehamna orientaux) par une étude thermobarométrique détaillée, confirme les prévisions chimiques. Ces conditions paroxysmales coincident avec celles du pic de pression. Cette coincidence, suggérant une relaxation thermique modérée, est attribuée à l'exhumation tectonique de la chaîne par extension crustale, dont les repercussions thermiques se sont traduites également par une décompression isotherme qui s'est achevée à basse pression dans le champ de stabilité de I'andalousite.
\end{abstract}

Abstract - In central Rehamna (Moroccan Hercynian Belt), the maximum crustal thickness was attained after the development of the Ouled Zedness Thrust, which emplaced the Jorf El Ahmar Formation (Eastern Rehamna) over the Skhour Nappe (central Rehamna) during a metamorphic event of intermediate $P / T$ reaching amphibolite-facies conditions. Later on, the El Broumi detachment caused the emplacement of a Devonian-Visean unit (El BroumiOuled Hassine and Lalla Tittaf Formations), previously metamorphosed in the low $T$ part of the amphibolite-facies, in the top of the structural ensemble during retrogressive metamorphic conditions. The structural and metamorphic study of the syn-peak mineral assemblages apparently suggests a similar tectonothermal evolution for the Skhour and Devonian-Visean formations. Nevertheless, mineral compositions in the developed metamorphic assemblages are indicative of higher metamorphic conditions in the first formation. Thermobarometric $P-T$ conditions of the regional thermal peak were calculated at $560^{\circ} \mathrm{C}$ and $9 \mathrm{kbar}$ in the Skhour Nappe, and $532^{\circ} \mathrm{C}$ and $7 \mathrm{kbar}$ in eastern Rehamna. These $P$ - $T$ conditions are also mainly coincident with those of the pressure peak. This suggests a very limited influence of decompressive thermal relaxation in the final tectonothermal evolution of the Rehamna Massif, interpreted as due to rapid exhumation of the belt as a result of syn-orogenic crustal extension. The syn-extensional part of the $P-T$ path shows slightly retrogressive decompression, which finally reached low pressure conditions in the andalusite stability field. 


\section{INTRODUCTION}

Le massif des Rehámna constitue une boutonnière de la mésèta marocaine (Fig. 1). Classiquement subdivisé en trois domaines, ce fragment de chaîne hercynienne comprend les Rehamna centraux et orientaux, affectés par un métamorphisme de moyenne $P / T$, et les Rehamna occidentaux peu à non métamorphiques (Fig. 2). Le métamorphisme barrovien affectant le domaine central du massif a été attribué par Jenny (1974), Hoeppiner et al. (1975) et Lagarde et Michard (1986) à des mouvements trans-currents constituant une anomalie cinématique dans la rampe frontale des mouvements chevauchants orientaux. Ce modèle présente néanmoins une difficulté majeure résidant dans le fait que ces décrochements ne peuvent engendrer un épaississement crustal susceptible de se traduire thermiquement par une évolution métamorphique barrovienne. Sougy (1976), Sougy et al. (1978), Corsini (1988) et Corsini et al. (1988) suggèrent ensuite des déplacements tangentiels qui ont amené l'ensemble du secteur central à s'engager dans des chevauchements sur les Rehamna occidentaux. L'étude structurale menée par la suite par Aghzer (1994) et Aghzer et Arenas (1995) dans la partie Nord du massif (Fig. 2), a permis de mettre en évidence une allochtonie majeure au sein même des Rehamna centraux, et un régime tectonique extensif, manifesté sous des conditions métamorphiques rétrogrades, qui est responsable de l'accordance finale des différents secteurs du massif.

Le pic thermique régional dans les Rehamna centraux et orientaux s'est établi durant les stades ultimes de la tectonique compressive et coincide essentiellement coincident avec le pic de pression (Aghzer et Arenas, 1995). La quantification des conditions $P$ - $T$ maximales et minimales du paroxysme thermique dans ces deux secteurs est l'objet de cette étude qui va intéresser exclusivement les assemblages minéralogiques des lithologies pélitiques. Ces conditions seront par la suite intégrées avec les données structurales afin de pouvoir préciser les traits fondamentaux de l'évolution tectono-métamorphique de ce fragment de chaîne de collision.

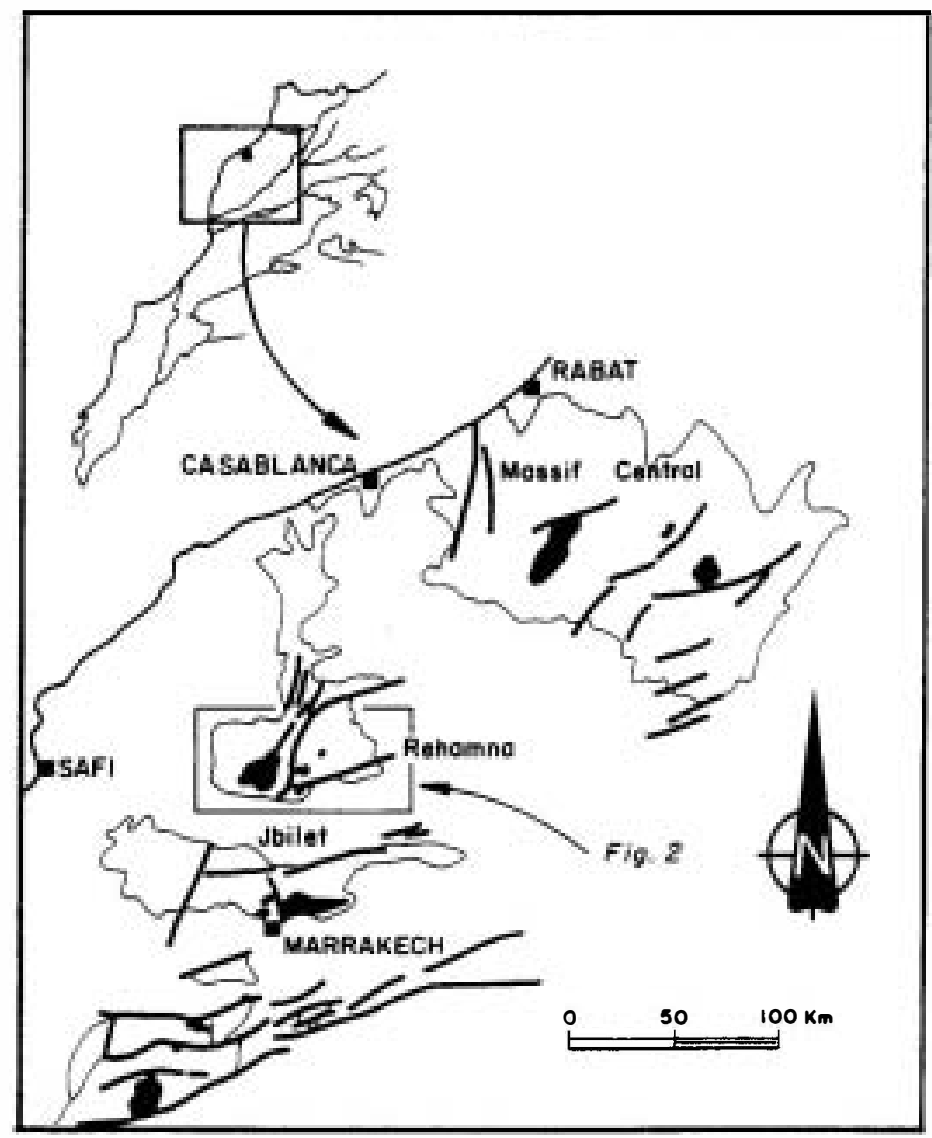

Figure 1. Localisation du massif des Rehamna dans un schéma géologique de la méséta marocaine. 


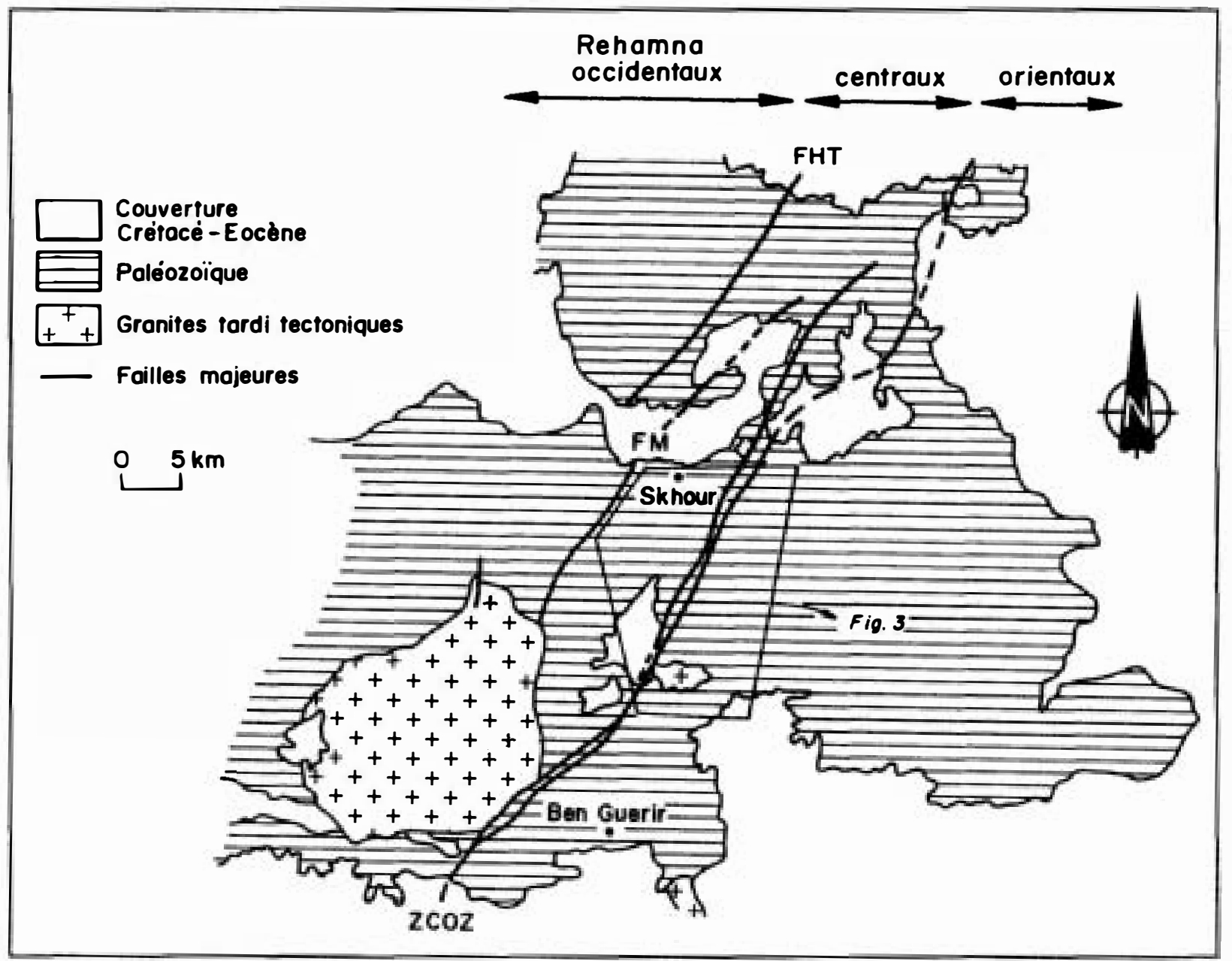

Figure 2. Carte géologique simplifiée du massif des Rehamna montrant les différents secteurs du massif et la localisation de la région étudiée. FHT: faille du haut Tarfa; FM: faille médiane; ZCOZ: zone de cisaillement d'Ouled Zedness; S: Skhour.

\section{CARACTERISTIQUES GEOLOGIQUES GENERALES}

Les Rehamna centraux sont principalement constitués par la nappe cambro-ordovicienne des Skhour, essentiellement composée de pélites, semipélites et quartzites (Fig. 3a), et dont la base est limitée par la zone de cisaillement de Kef EI Mouneb (ZCKM) (Fig. 3), correspondant au toit fortement cisaillé de I' auto ou para-autochtone (formation de Kef El Mouneb) qui est formé de matériaux conglomératiques à intercalations de niveaux semipélitiques ferrugineux (Piqué, 1972), datés par Corsini (1988) du Dévonien. Cette limite tectonique, initialement de type chevauchement, est réactivée postérieurement au cours de l'extension crustale en faille normale ductile (Aghzer, 1994; Aghzer et Arenas, 1995).

Le secteur central est séparé du secteur oriental sus-jacent par une zone de cisaillement de nature complexe (zone de cisaillement d'Ouled Zedness ou ZCOZ), dont la limite tectonique orientale est de type chevauchement
(Jenny, 1974; Hoeppfner et al., 1975; Corsini et al., 1988; Aghzer, 1994; Aghzer et Arenas, 1995), alors que l'occidentale est de type faille normale ductile (Aghzer, 1994; Aghzer et Arenas, 1995).

Les matériaux orientaux s' organisent en trois formations lithostratigraphiques qui sont du bas vers le haut (Fig. 3a):

i) la formation cambro-ordovicienne de Jorf El Ahmar, formée essentiellement de pélites et quartzites (Jenny, 1974);

ii) la formation dévonienne d'El Broumi-Ouled Hassine (Aghzer, 1994), correspondant à une série pélitique à intercalations de quartzites et métabasites; et

iii) la formation de Lalla Tittaf (Jenny, 1974), d'âge présumé Tournaisien à Viséen (Aghzer, 1994), comprenant des métapélites et semipélites à intercalations de métabasites, orthogneiss, calcschistes et marbres.

Les deux premières formations sont séparées par la zone de cisaillement d'El Broumi (ZCEB), 


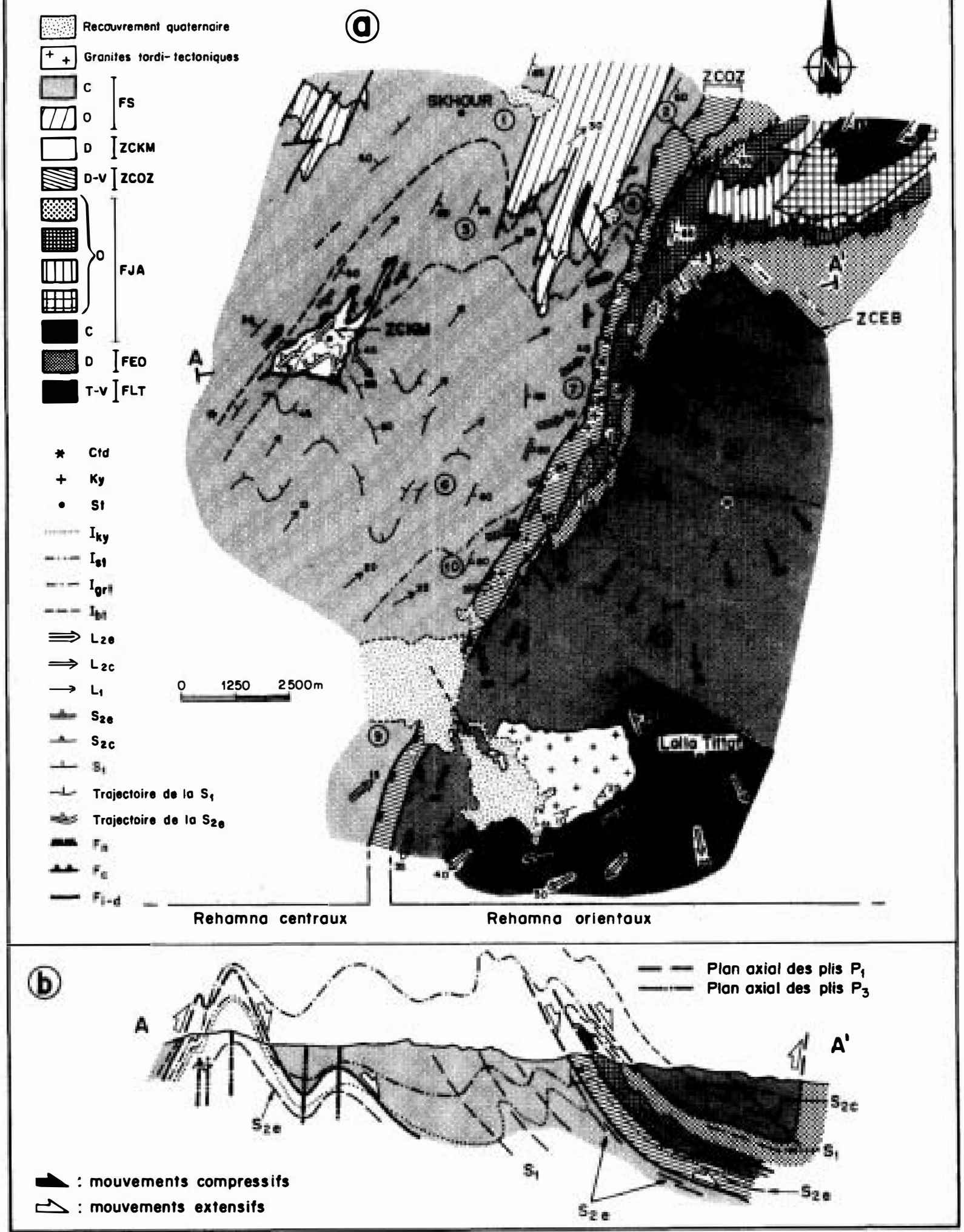

Figure 3. (a) Carte géologique simplifiée du secteur étudié. C: Cambrien; O: Ordovicien; D: Dévonien; D-V: Dévonien à Viséen; T-V: Toumaisien à Viséen; FS: formation de Skhour; ZCKM: zone de cisaillement de Kef EI Mouneb; ZCOZ: zone de cisaillement d'Ouled Zedness; FJA: Formation de Jorf El Ahmar; FEO: formation d'El Broumi-Ouled Hassine; FLT: formation de Lalla Tittaf. $K y$ : localités du disthène; St: localités de la staurotide; Ctd: localités du chloritoïde; $I_{k y}$ : isograde du disthène; $I_{s i}$ isograde de la staurotide; $l_{g r t}$ isograde du grenat; $l_{b r}$ isograde de la biotite; $L_{2 e}$ : linéation d'étirement associée à la déformation $D_{2 e^{j}} L_{2 c}$ : linéation d'étirement associée à la déformation $D_{2 c}: L_{1}$ : linéation d'étirement associée à la déformation $D_{, i} S_{2 c}$ : plan de schistosité de la déformation $D_{2 c} ; S_{2 e}$ : plan de schistosité de la déformation $D_{2 s}: S_{1}$ : plan de schistosité de la déformation $D_{1}$; $F_{n}$ : faille normale ductile; $F_{c}$ : chevauchement; $F_{i-\sigma}$ failles inverses ou décrochements. Les numéros entourés par des cercles indiquent la localisation des échantillons analysés. (b) Coupe géologique. 
à caractère de faille normale ductile (Aghzer, 1994; Aghzer et Arenas, 1995; Fig. 3).

\section{CARACTERISTIQUES STRUCTURALES}

Les matériaux impliqués dans la déformation hercynienne ont enregistré trois phases de déformation $D_{1}, D_{2}$ et $D_{3}$.

La déformation $D_{1}$ a engendré des plis d'ampleur kilométrique (Fig. 3), définissant régionalement dans les formations des Skhour et de Jorf El Ahmar des anticlinaux de direction axiale NE-SO, à vergence NO et à faible plongement axial vers le NE (Jenny, 1974; Hoeppfner et al., 1975). Ces plis développent une schistosité de plan axial $S_{1}$ à caractère régional.

Deux régimes tectoniques caractérisent la déformation $D_{2}$ : un régime compressif précoce $\left(D_{2 c}\right)$ auquel succède un autre extensif tardif $\left(D_{2 e}\right)$. La ZCKM et la limite tectonique orientale de la ZCOZ se sont manifestées au cours de la déformation $D_{2 c}$ tout en assurant les chevauchements, dirigés vers I'O à NO, de la formation auto ou para-autochtone de Kef El Mouneb par la formation des Skhour (Aghzer, 1994; Aghzer et Arenas, 1995), et des Rehamna centraux par la formation de Jorf El Ahmar (Fig. 3). Les structures $D_{2 c}$ correspondent à des plis $\left(P_{2}\right)$ isoclinaux de taille métrique à millimétrique, à faible plongement axial vers le $\mathrm{N}$ à $\mathrm{NE}$ et à déversement net vers l'O à NO. Ces plis développent une schistosité de plan axial $\mathrm{S}_{2}\left(\mathrm{~S}_{2}\right.$ compressive ou $S_{2 c}$ ), pénétrative qui évolue souvent vers une foliation mylonitique. Ces structures compressives ont été également rencontrées dans des couloirs étroits de déformation syn-schisteuse, localisés dans les formations cambro-ordoviciennes du massif (formations des Skhour et de Jorf EI Ahmar), juste à leurs contacts avec les zones de cisaillement. L'accordance des différents secteurs du massif, déjà initiée au cours des chevauchements, s'achève par la tectonique extensive qui s'est manifestée dans la ZCKM, réactivée à ce stade de la déformation hercynienne en faille de détachement, et la limite tectonique occidentale de la $\mathrm{ZCOZ}$, qui ont respectivement assuré la mise en place finale de la nappe des Skhour sur la formation de Kef El Mouneb et de la formation de Jorf El Ahmar sur le secteur central (Fig. 3) (Aghzer, 1994; Aghzer et Arenas, 1995). La tectonique extensive a conditionné également la mise en place du bloc dévono-viséen oriental (formations d'El Broumi-Ouled Hassine et de Lalla Tittaf) au sommet de l'édifice orogénique. Les structures régionales de ce bloc relèvent de la déformation $D_{2 c}$ : il s'agit de plis $P_{2}$, développant une schistosité de plan axial $\mathrm{S}_{2 \mathrm{c}}$ à caractère régional, et montrant les mêmes caractéristiques géométriques que celles décrites précédement. Les plans de schistosité $S_{1}$, définis par des associations épizonales à chlorite ou à chloritoïde, sont localement épargnés des processus de transposition dans les charnières $P_{2}$. Les failles de détachement ont engendré une schistosité $\mathrm{S}_{2} \quad\left(\mathrm{~S}_{2}\right.$ extensive ou $\left.\mathrm{S}_{2 \mathrm{e}}\right)$ extrêmement pénétrative, à caractère souvent mylonitique, qui a presque complètement oblitéré les structures antérieures. Ces fabriques planaires extensives définissent également des bandes étroites, plus ou moins continues, de déformation synschisteuse limitant les parties basales et/ou sommitales des différentes formations du massif à leurs contacts avec les failles de détachement. Les plans de schistosité $S_{2 e}$ portent des linéations d'étirement plongeantes vers le NE dans la ZCKM et la limite tectonique occidentale de la ZCOZ, et vers le SE dans la ZCEB (Fig. 3a). Les critères de cisaillement selon ces fabriques linéaires indiquent une variation cinématique des mouvements avec la profondeur (Aghzer et Arenas, 1995): le déplacement tectonique vers le NE des formations cambro-ordoviciennes s'opère, par contre, vers le SE dans le bloc dévono-viséen oriental.

Les structures $D_{1}$ et $D_{2}$ sont ensuite affectées par un plissement et des décrochements tardifs de troisième phase tectonique (Fig. 3). Le plissement $P_{3}$ est à l'origine de la configuration géométrique du massif en antiforme-synforme (Fig. 3): dans le secteur central, il a donné lieu à un anticlinal coaxial aux plis $P_{1}$ de la formation des Skhour (Fig. 3), droit à déversé vers le NO, à faible plongement axial vers le $\mathrm{NE}$, alors que dans le secteur oriental, il a engendré un synclinal de direction N140, à déversement modéré vers le SO (Fig. 3b) et à fort plongement axial vers le SE. Quant aux décrochements, ils s'organisent en quatre familles généralement dextres (Fig. 3a): deux familles conjuguées N140-170 dextre et N80-90 senestre, une troisièeme famille orientée $\mathrm{NE}-\mathrm{SO}$ et enfin une dernière orientée $\mathrm{N}$-S.

\section{METAMORPHISME}

Les métapélites et semipélites montrent, selon une coupe $\mathrm{N}-\mathrm{S}$, une zonation métamorphique d'affinité barrovienne allant de la zone de la chlorite jusqu'à la zone de la staurotide (formation des Skhour et secteur oriental) ou celle du disthène (ZCKM et ZCOZ; Fig. 3 ). Le 
Table 1. Assemblages minéralogiques de la séquence métamorphique barrovienne dans I'ensemble du secteur étudié

\begin{tabular}{|c|c|}
\hline Zone de la chlorite & $\begin{array}{l}C h l+M s+Q t z \pm S t p \pm P l+\| m \pm R t \\
C t d+M s+C h l+Q t z+\| m(F S)\end{array}$ \\
\hline Zone de la biotite & $\mathrm{Bt}+\mathrm{Ms}+\mathrm{Qtz} \pm \mathrm{Chl} \pm \mathrm{Stp} \pm \mathrm{Pl}+\mathrm{llm} \pm \mathrm{Rt}$ \\
\hline \multirow[t]{2}{*}{ Zone du grenat } & $\mathrm{Grt}+\mathrm{Bt}+\mathrm{Ms}+\mathrm{Qtz} \pm \mathrm{Chl} \pm \mathrm{Pl}+\| \mathrm{lm} \pm \mathrm{Rt}$ \\
\hline & $\mathrm{Ctd}+\mathrm{Ms}+\mathrm{Chl}+\mathrm{Qtz}+\mathrm{Pl}+\mathrm{IIm}+\mathrm{Rt}(\mathrm{ZCOZ})$ \\
\hline \multirow[t]{2}{*}{ Zone de la staurotide } & $\mathrm{St}+\mathrm{Grt}+\mathrm{Bt}+\mathrm{Ms}+\mathrm{Qtz}+\mathrm{Pl} \pm \mathrm{Chl}+\| \mathrm{Im} \pm \mathrm{Rt}$ \\
\hline & $\mathrm{St}+\mathrm{Ctd}+\mathrm{Ms}+\mathrm{Qtz}+\mathrm{Pl} \pm \mathrm{Chl}+\| \mathrm{m} \pm \mathrm{Rt}(\mathrm{ZCOZ}$ et $\mathrm{FEO})$ \\
\hline Zone du disthène & $\begin{array}{l}\mathrm{St}+\mathrm{Bt}+\mathrm{Ms}+\mathrm{Qtz}+\mathrm{Pl} \pm \mathrm{Grt}+\| \mathrm{lm} \pm \mathrm{Rt}(\mathrm{ZCKM} \text { et } \mathrm{ZCOZ}) \\
\mathrm{Ky}+\mathrm{Ms}+\mathrm{Qtz}+\mathrm{Pl} \pm \mathrm{St} \pm \mathrm{Bt} \pm \mathrm{Chl}+\| \mathrm{m} \pm \mathrm{Rt} \quad(\mathrm{ZCKM} \text { et } \mathrm{ZCOZ})\end{array}$ \\
\hline
\end{tabular}

Les abréviations des minéraux sont celles proposées par Kretz (1983). Les autres abréviations sont identiques à celles de la Figure 3.

chloritoïde montre une répartition sporadique qui relève probablement des variations de la teneur en alumine dans les métapélites: son occurrence est limitée à la zone de la chlorite de la formation des Skhour, à la zone de la staurotide de la formation d'El Broumi-Ouled Hassine et aux zones du grenat et de la staurotide de la $\mathrm{ZCOZ}$ (Fig. 3 et Table 1). Les différents assemblages minéralogiques développés en fonction de la zonation métamorphique barrovienne sont reportés dans la Table 1 .

\section{Relation entre le métamorphisme et la déformation}

Formation des Skhour et Rehamna orientaux

Les formations cambro-ordoviciennes du massif sont affectées par des plans de schistosité $S$, à caractère régional (Fig. $3 a$ ). Localement, à leurs contacts avec les failles de détachement, ces formations sont affectées par des plans de schistosité $S_{2 c}$ et $S_{2 e}$. Les fabriques planaires régionales des formations dévono-viséennes relèvent par contre de la déformation $D_{2 c}$ (Fig. 3). Des plans de schistosité $S_{1}$ y sont localement préservés dans les charnières des microplis $P_{2}$. La base de la formation d'El Broumi a enregistré les effets de la déformation $D_{2 e}$ dont les plans $d^{\prime}$ anisotropie $S_{2 e}$ délimitent un couloir de déformation intense localisé au contact de la ZCEB (Fig. 3).

La relation temporelle entre le métamorphisme et la déformation dans les formations précitées est reportée dans la Figure 4.

Le stipnomélane, la chlorite, la muscovite et la biotite participent à la définition des plans de schistosité $S_{1}, S_{2 c}$ et $S_{2 e}$ des métapélites et semipélites des zones de la chlorite et de la biotite. Par leurs dispositions parfois discordantes sur la schistosité $S_{2}\left(S_{2 c}\right.$ et $\left.S_{2 e}\right)$, ces minéraux définissent également une troisième génération post-schisteuse. La blastèse post- $S_{2} d u$ stipnomélane et de la biotite est cependant limitée par rapport à celle de la chlorite qui se forme souvent à leurs dépens. Dans la formation des Skhour, les fabriques planaires $S_{1}$ sont localement soulignés dans la zone de la chlorite par des prismes subidiomorphes de chloritoïde.

A part le stipnomélane qui disparait à partir de la partie inférieure de la zone de la biotite, et de la chlorite qui fait défaut dans les assemblages progrades de la zone de la staurotide, les différentes générations des minéraux précités se retrouvent dans les roches de forts grades. Dans ces roches, la croissance du grenat est contemporaine de la déformation $D_{1}$ et $D_{2 c}$. Ce minéral est toujours moulé par la schistosité $S_{1}$ ou $S_{2 c}$ qui s'y prolonge sous forme de schistosité interne ( $\mathrm{Si}$ ) adoptant des géométries systématiquement variables depuis l'isograde du grenat jusqu'à la zone de la staurotide: sa croissance est tardi-schisteuse au niveau de l'isograde $\mathrm{Gr}(+)$, où il englobe une Si linéaire et parallèle à la Se, alors qu'elle est syn-schisteuse dans la zone de la staurotide et la partie inférieure de la zone du grenat où sa Si adopte des figures de rotation syn-cinématique (Fig. 5). Le grenat continue de se développer au cours de la déformation $D_{2 e}$; sa $S i$, passant en continuité avec les plans de schistosité externe (Se), adopte une géométrie sigmoïdale suggérant une croissance syn-schisteuse. Les bords des grenats de l'ensemble des générations précitées sont le plus souvent rétrogradés en biotite et/ou chlorite. La staurotide entretient globalement les mêmes relations microstructurales avec les fabriques planaires $S_{1}, S_{2 c}$ et $S_{2 e}$ que celles décrites dans le cas du grenat. Néanmoins, sa croissance s'est initiée après celle de ce dernier qu'elle englobe sous forme d'inclusions plus ou moins partielles, 


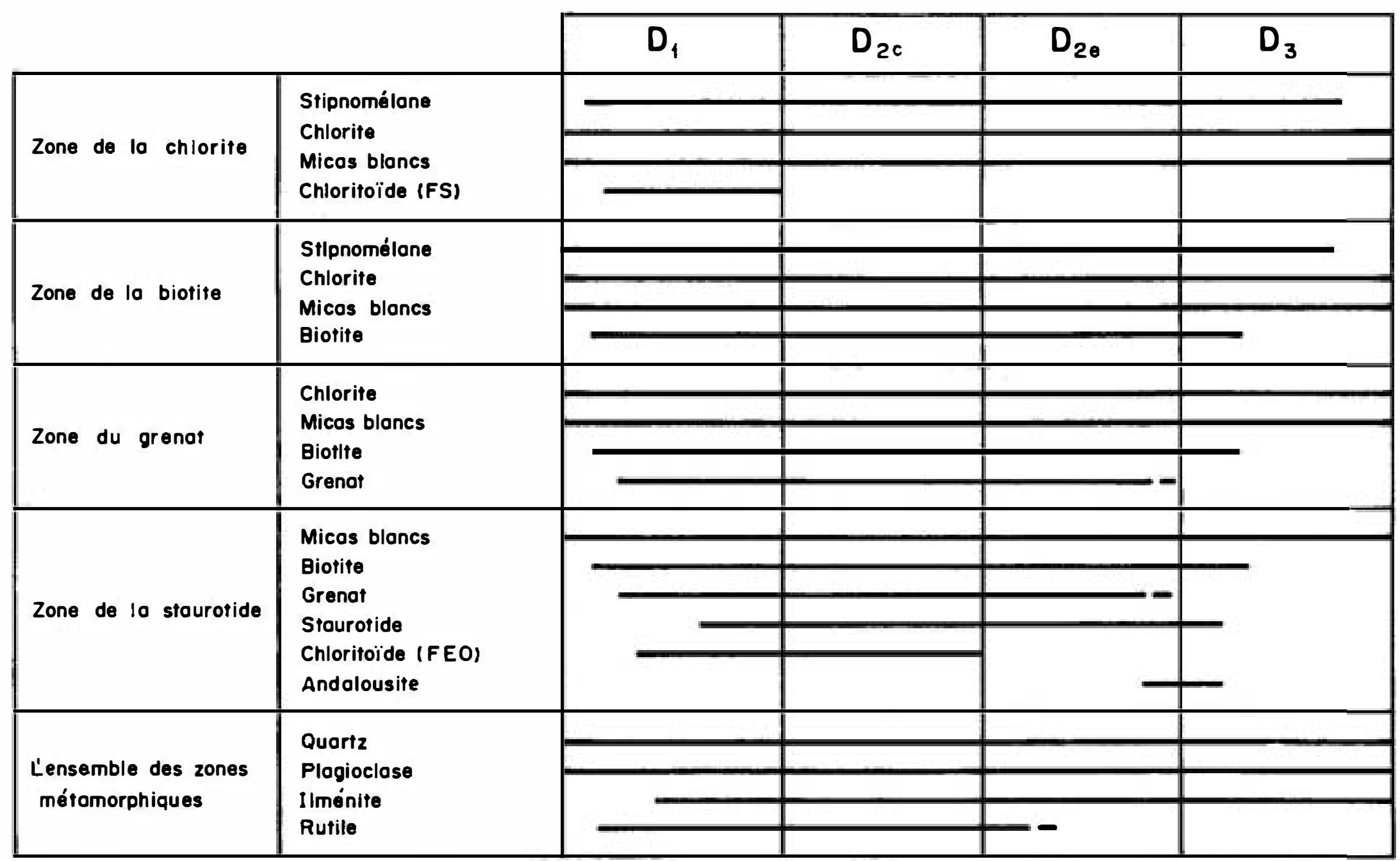

Figure 4. Relation déformation-métamorphisme dans la formation de Skhour et le secteur oriental. FS: formation de Skhour; FEO: formation d'El Broumi-Ouled Hassine. 


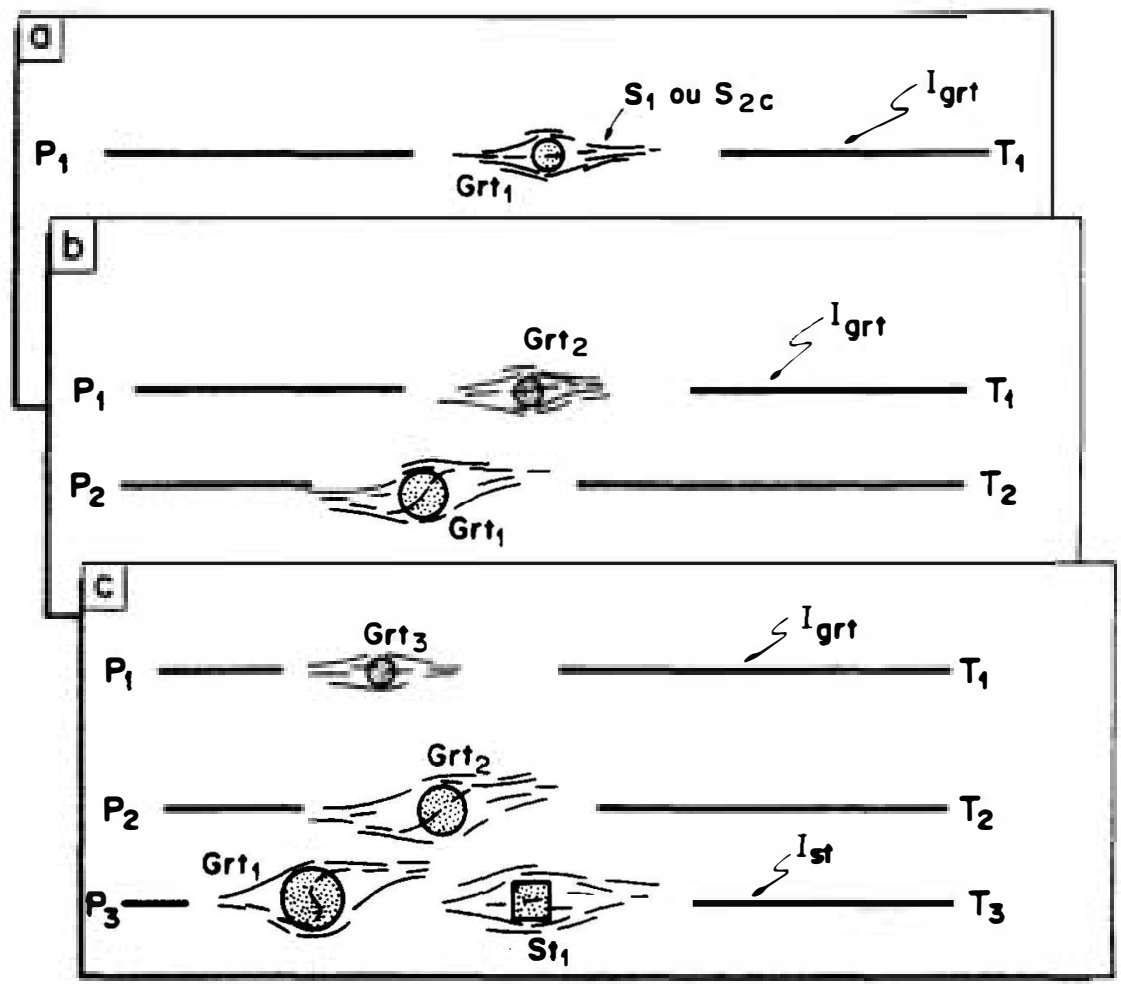

Figure 5. Stades successifs (a), (b) et (c) de croissance du grenat en fonction de la profondeur au cours de l'incrément de la déformation $D$, ou $D_{2 c}$. Les isothermes sont supposées confondues avec les isogrades d'une part, et parallèles aux isobares d'autre part. Grt: grenat; St: staurotide; $I_{g r}$ : isograde du grenat; $S_{r}$ : schistosité de première phase tectonique; $S_{2 c}$ : schistosité associée à la déformation $D_{2}$ compressive; $P_{1}, P_{2}$ et $P_{3}$ : isobares avec $P_{3}>P_{2}>P_{1} ; T_{1}, T_{2}$ et $T_{3}$ : isothermes avec $T_{3}>T_{2}>T_{1}$.

et continue de s'opérer postérieurement à la définition des plans $S_{2 e}$. Des transformations rétrogrades en muscovite, chlorite et/ou biotite affectent souvent ses bords. II est à remarquer que les assemblages syn- $D_{1}$ à staurotide, à grenat ou à biotite $n$ 'ont jamais été rencontrés dans les formations dévono-viséennes orientales et les zones de cisaillement, où les plans $S_{1}$ ne sont définis que par des associations épizonales à chlorite ou, localement, à chloritoïde. L'andalousite se développe en grande partie en réponse aux perturbations locales de la structure thermique régionale par la mise en place des granitoïdes tardi-tectoniques à proximité de la ZCEB et la ZCOZ. Ce silicate d'alumine est essentiellement tardi-schisteux; il est moulé par la schistosité $\mathrm{S}_{2 \mathrm{e}}$ et englobe une Si continue avec la Se, et orientée parallèlement à elle. II montre également des dispositions aléatoires sur la schistosité $\mathrm{S}_{2 \mathrm{e}^{\prime}}$ indiquant une croissance postcinématique.

Le rutile et l'ilménite contribuent à l'impression des plans $S_{1}, S_{2 c}$ et $S_{2 e}$ de l'ensemble des zones métamorphiques. Sur les bords du premier minéral, on décèle souvent un début de transformation en ilménite.

\section{Failles de détachement}

La relation entre le métamorphisme et la déformation est illustrée dans la Figure 6.

Les fabriques planaires $S_{2 e}$ des détachements évolue souvent vers une foliation mylonitique qui a généralement oblitéré les structures antérieures. Localement, des plis $\mathrm{P}_{2}$ à schistosité de plan axial $\mathrm{S}_{2 \mathrm{c}}$ sont épargnés de la composante de cisaillement. Les plans $S_{1}$ microplissés au niveau des microcharnières $P_{2}$ et les plans $S_{2 c}$ sont définis par des assemblages épizonaux à chlorite, muscovite et, localement à chloritoïde (cas de la ZCOZ). Les mêmes assemblages syn et post- $S_{2 \mathrm{e}}$ précités dans la formation des Skhour et dans le secteur oriental se retrouvent dans les failles de détachement où ils entretiennent les mêmes relations microstructurales décrites précédement avec la $S_{2 \mathrm{e}}$. II est cependant à noter que la croissance du grenat et de la staurotide s'est initiée durant les stades tardifs de la déformation $D_{2 c^{\prime}}$ comme en attestent leur Se $\left(\mathrm{S}_{2 \mathrm{e}}\right)$ parfois discordante sur la Si. Les fabriques planaires extensives sont également définies par des assemblages à disthène dont l'occurrence est restreinte aux micaschistes de la ZCKM et la ZCOZ. La Si sigmoïdale de ce silicate d'alumine, 


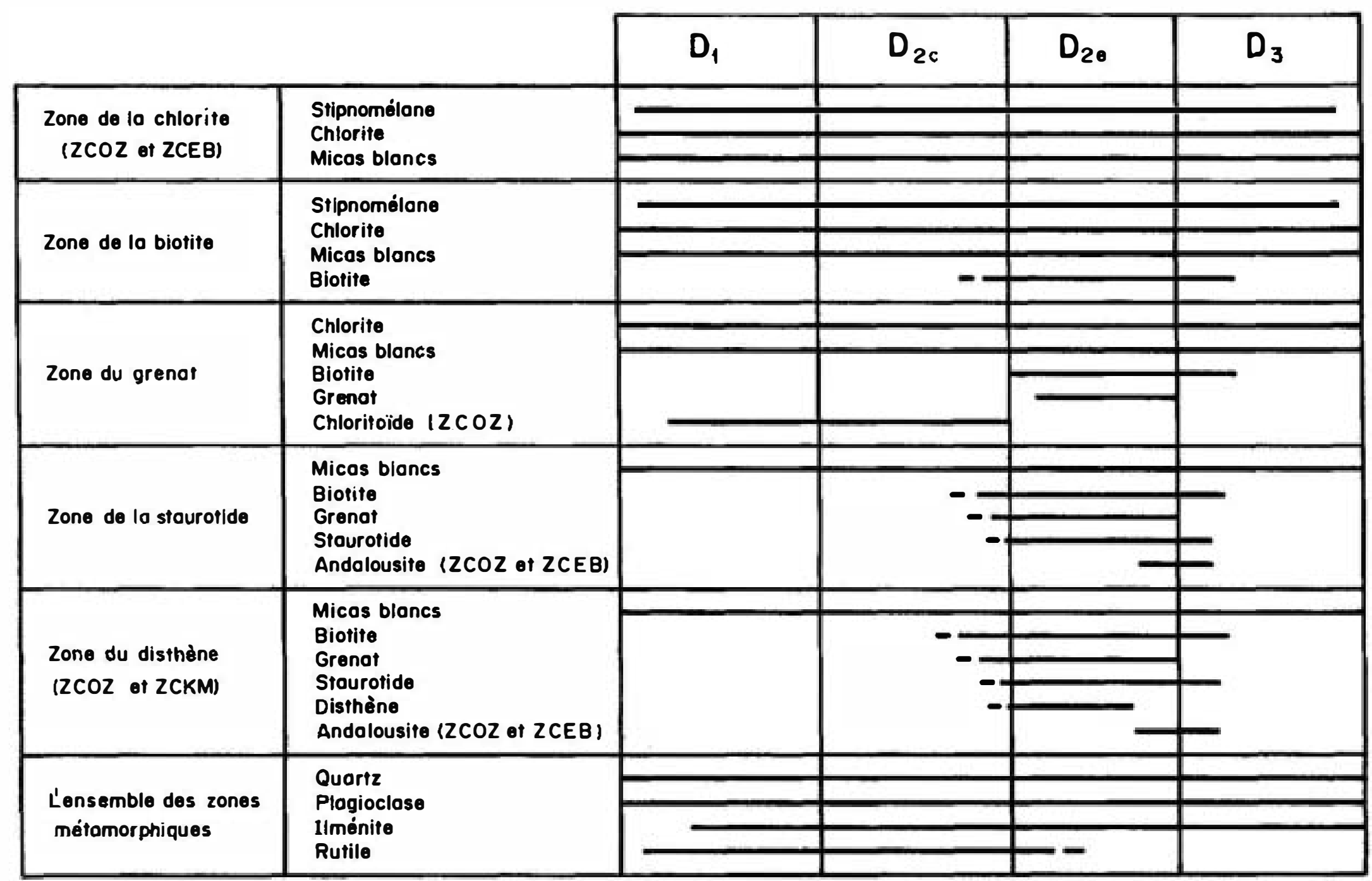

Figure 6. Relation temporelle entre le métamorphisme et la déformation dans les failles de détachement. ZCOZ: zone de cisaillement de Ouled Zedness; 2CKM: zone de cisaillement de Kef El Mouneb; ZCEB: zone de cisaillement d'El Broumi. 
étant plus ou moins tronquée par les plans $S_{2 e^{\prime}}$ suggère une croissance pré à syn-schisteuse. Le disthène a été également rencontré dans des filons de quartz concordants avec la foliation régionale. Ses bords sont le plus souvent rétrogradés en muscovite.

La schistosité $S_{2 e}$ des failles de détachement évolue souvent vers une foliation mylonitique par reduction de la taille des grains des roches. Elles est, par conséquent, associée à une évolution métamorphique rétrograde qui devrait être, néanmoins, assez modérée puisqu'elle est, en partie, accompagnée par les mêmes assemblages métamorphiques syn- $D_{2 c}$.

\section{Isogrades}

Les zones métamorphiques sont limitées par des isogrades plissés par la troisième phase tectonique (Fig. 3). A partir de l'étude de la relation déformation-blastèse, il s'avère que la croissance ultime de la chlorite, de la biotite et de la staurotide s'est prolongée jusqu' aux stades précoces de la déformation $D_{3}$. Ce fait explique bien la discordance des isogrades des minéraux index en question sur les tracés cartographiques de la ZCOZ et la ZCEB, à jeux contemporains de la déformation $D_{2}$ (Fig. 3a). L'isograde du grenat, quant à lui, est par contre diachronique en ce sens que l'âge relatif de sa définition par rapport aux différents plans de schistosité est variable à travers le secteur étudié: sa définition est principalement syn- $D$, dans les formations cambro-ordoviciennes, alors qu' elle est syn- $D_{2 c}$ et/ou $D_{2 e}$ dans les zones de cisaillement et dans le bloc dévono-viséen oriental. Cet isograde, bien qu'étant anté- $D_{3}$, recoupe les tracés cartographiques des zones de cisaillement (Fig. 3a). Cette relation géométrique paradoxale est attribuée au caractère diachronique de l'isograde en question: différentes générations d'isogrades $\mathrm{Gr}(+)$ se recoupent au niveau et près des zones de cisaillement, tout en donnant lieu à un isograde résultant, virtuellement discordant sur les tracés cartographiques des contacts tectoniques (Fig. 3a).

Les zones métamorphiques ont subi une forte condensation qui s'est traduite par les distances relativement faibles, de l'ordre de 5 à $6 \mathrm{~km}$, séparant, hors des zones de cisaillement, la zone de la chlorite de celle de la staurotide (Fig. 3a). Dans la ZCKM et la ZCOZ, la coincidence locale de l'isograde St( +) avec l'isograde $\mathrm{Ky}(+)$ ) (Fig. 3) rend compte de cette condensation, qui est particulièrement spectaculaire dans la première zone de cisaillement où la distance séparant la zone de la biotite de celle du disthène est de
250 à $300 \mathrm{~m}$ (Fig. 3). Selon Michard (1968), ce resserrement des isogrades est attribué à un gradient thermique fort, de $50^{\circ} \mathrm{C} \mathrm{km}^{-1}$, imputé à la mise en place tardive des granitoïdes. L'existence d'un tel gradient implique que la relaxation thermique est assez effective pour aboutir au développement d'assemblages métamorphiques progrades de haute température et basse pression. L'absence d'assemblages métamorphiques de fort degré (migmatites et assemblages à sillimanite) au niveau et hors du secteur étudié (Jenny, 1974; Aghzer, 1994) et la faible abondance des intrusions granitiques suggèrent que les influences thermiques des granites sont limitées. Ce caractère modéré de la relaxation thermique est attribué à l'exhumation rapide de la chaîne par la tectonique extensive qui est en grande partie à l'origine de l'absence d'une séquence zonéographique barrovienne complète dans la section crustale exposée à la surface d'érosion dans le massif. La condensation des zones métamorphiques, de par le fait qu'elle est particulièrement intense au niveau et près des failles de détachement, est attribuée à la tectonique extensive qui a donné lieu à un amincissement crustal considérable. Cet amincissement peut être apprécié par les estimations barométriques (voir Table 6) réalisées dans les Rehamna orientaux où les différences de pression entre la zone de la biotite et celle de la staurotide revèlent, compte tenu de la distance actuelle séparant ces zones, un déficit de charge minimal de 11 à $12 \mathrm{~km}$.

\section{MINERALOGIE}

Des analyses à la microsonde ont porté sur les phases minérales des formations des Skhour, d'El Broumi-Ouled Hassine et de Lalla Tittaf. Bien que $n$ 'ayant principalement intéressé que les assemblages syn- $D_{2 c}$, ces analyses ont été également réalisées sur les phases minérales syn$D_{1}$ de la formation des Skhour. Les assemblages à chlorite des formations orientales $n$ 'ont pas fait l'objet d'analyses, et ce en raison de leur interêt thermobarométrique extrêmement limité.

\section{Grenat}

Les grenats syn- $D_{1}$ de l'isograde $\mathrm{Gr}(+)$ de la formation des Skhour, riches en spessartine et pauvres en pyrope, sont de type pyralspite (Table 2). La composition moyenne de leurs coeurs $\left(\mathrm{Alm}_{59.27} \mathrm{Sps}_{28.37} \operatorname{Prp}_{5.55} \mathrm{Grs}_{6.81}\right)$ est presque similaire à celle de leurs bords $\left(\mathrm{Alm}_{59.94} \mathrm{Sps}_{27.84}\right.$ $\operatorname{Prp}_{5.43}$ Grs $_{6.79}$ ). Les grenats syn-D, de la zone de la staurotide et de la partie inférieure de la zone 
Table 2. Analyses chimiques représentatives des grenats syn- $D_{1}$ et $s y n-D_{2 c}$ des métapélites et semipélites de la formation de Skhour (FS) et des formations d'El Broumi-Ouled Hassine et de Lalla Tittaf (FEOL)

\begin{tabular}{|c|c|c|c|c|c|c|c|c|c|c|c|c|}
\hline & \multicolumn{8}{|c|}{ FS } & \multicolumn{4}{|c|}{ FEOL } \\
\hline & \multicolumn{4}{|c|}{ Syn $D_{1}$} & \multicolumn{8}{|c|}{ Syn $D_{2 c}$} \\
\hline & 6G1.B & 6G1.C & 9G1.B & 9G1.C & 7G1.B & 7G1.C & 10G1.B & 10G1.C & 8G1.B & 8G1.C & 11G1.B & $11 \mathrm{G} 1 . \mathrm{C}$ \\
\hline $\mathrm{SiO}_{2}$ & 36.80 & 36.31 & 37.27 & 37.33 & 37.05 & 37.19 & 36.89 & 36.44 & 37.14 & 36.76 & 37.54 & 36.55 \\
\hline $\mathrm{TiO}_{2}$ & 0.23 & 0.02 & 0.06 & 0.03 & 0.04 & 0.08 & 0.08 & 0.13 & 0.05 & 0.19 & 0.03 & 0.09 \\
\hline $\mathrm{Al}_{2} \mathrm{O}_{3}$ & 20.11 & 20.15 & 21.69 & 20.48 & 20.14 & 20.36 & 20.28 & 20.16 & 20.71 & 19.94 & 20.90 & 20.28 \\
\hline $\mathrm{FeO}$ & 26.71 & 26.17 & 35.00 & 33.48 & 35.81 & 31.95 & 35.55 & 33.69 & 31.19 & 21.00 & 35.64 & 30.09 \\
\hline $\mathrm{MnO}$ & 11.62 & 11.73 & 0.48 & 4.55 & 3.42 & 7.14 & 1.14 & 4.23 & 6.16 & 17.76 & 2.49 & 8.50 \\
\hline $\mathrm{MgO}$ & 1.25 & 1.37 & 2.65 & 1.76 & 1.41 & 1.05 & 2.54 & 1.97 & 0.97 & 0.55 & 2.18 & 1.00 \\
\hline $\mathrm{CaO}$ & 2.60 & 2.52 & 3.15 & 4.15 & 2.31 & 2.51 & 2.01 & 2.44 & 3.04 & 2.99 & 2.49 & 4.14 \\
\hline Total & 9.31 & 98.27 & 100.35 & 101.81 & 100.20 & 100.30 & 98.50 & 99.12 & 99.26 & 99.19 & 101.47 & 100.65 \\
\hline \multicolumn{13}{|c|}{ Cations calculés sur la base de 12 oxygênes } \\
\hline Si & 3.02 & 3.00 & 2.98 & 2.97 & 3.02 & 3.03 & 3.03 & 2.99 & 3.04 & 3.03 & 3.00 & 2.96 \\
\hline $\mathrm{Ti}$ & 0.01 & - & - & $=$ & - & $\cdot$ & . & 0.01 & - & 0.01 & + & 0.01 \\
\hline Al & 1.96 & 1.97 & 2.05 & 1.93 & 1.95 & 1.98 & 2.02 & 1.94 & 2.05 & 1.97 & 1.97 & 1.93 \\
\hline $\mathrm{Fe}^{2+}$ & 1.83 & 1.81 & 2.34 & 2.26 & 2.44 & 2.18 & 2.44 & 2.30 & 2.14 & 1.45 & 2.38 & 2.04 \\
\hline Mn & 0.81 & 0.82 & 0.03 & 0.31 & 0.24 & 0.49 & 0.08 & 0.29 & 0.43 & 1.24 & 0.17 & 0.58 \\
\hline $\mathrm{Mg}$ & 0.15 & 0.17 & 0.32 & 0.21 & 0.17 & 0.13 & 0.31 & 0.24 & 0.12 & 0.07 & 0.26 & 0.12 \\
\hline $\mathrm{Ca}$ & 0.23 & 0.22 & 0.27 & 0.35 & 0.20 & 0.22 & 0.18 & 0.21 & 0.27 & 0.26 & 0.21 & 0.36 \\
\hline Total & 8.01 & 7.99 & 7.99 & 8.03 & 8.02 & 8.03 & 8.03 & 7.98 & 8.05 & 8.03 & 7.99 & 8.00 \\
\hline Alm & 60.65 & 59.59 & 79.11 & 70.75 & 79.85 & 72.17 & 81.15 & 75.00 & 72.45 & 47.95 & 78.63 & 64.15 \\
\hline Sps & 26.72 & 27.35 & 1.10 & 10.32 & 7.81 & 16.34 & 2.64 & 9.81 & 14.49 & 41.07 & 5.62 & 19.66 \\
\hline Prp & 5.06 & 5.62 & 10.67 & 7.02 & 5.67 & 4.23 & 10.33 & 8.04 & 4.01 & 2.24 & 8.65 & 4.07 \\
\hline Grs & 6.86 & 5.99 & 9.12 & 11.90 & 6.68 & 7.26 & 5.88 & 7.15 & 9.05 & 8.75 & 7.10 & 12.11 \\
\hline
\end{tabular}

6, 7 et 8: zone du grenat; 9, 10 et 11: zone de la staurotide; B: bords; C: coeurs.

(a)

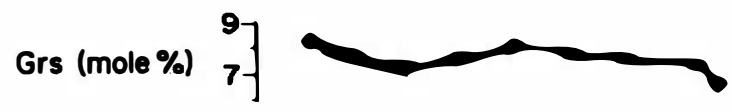

Prp (mole \%) $\left.{ }^{7}\right]$

Sps (mole \%) 29

Alm $\left(\right.$ mole\%) $\left.{ }_{59}^{61}\right]$
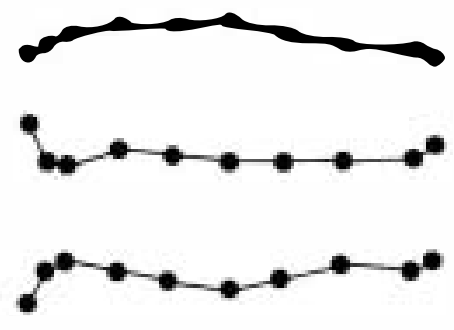

B

$02040 \quad 60 \quad 80 \quad 100120$

Distonce $(\mu \mathrm{m})$

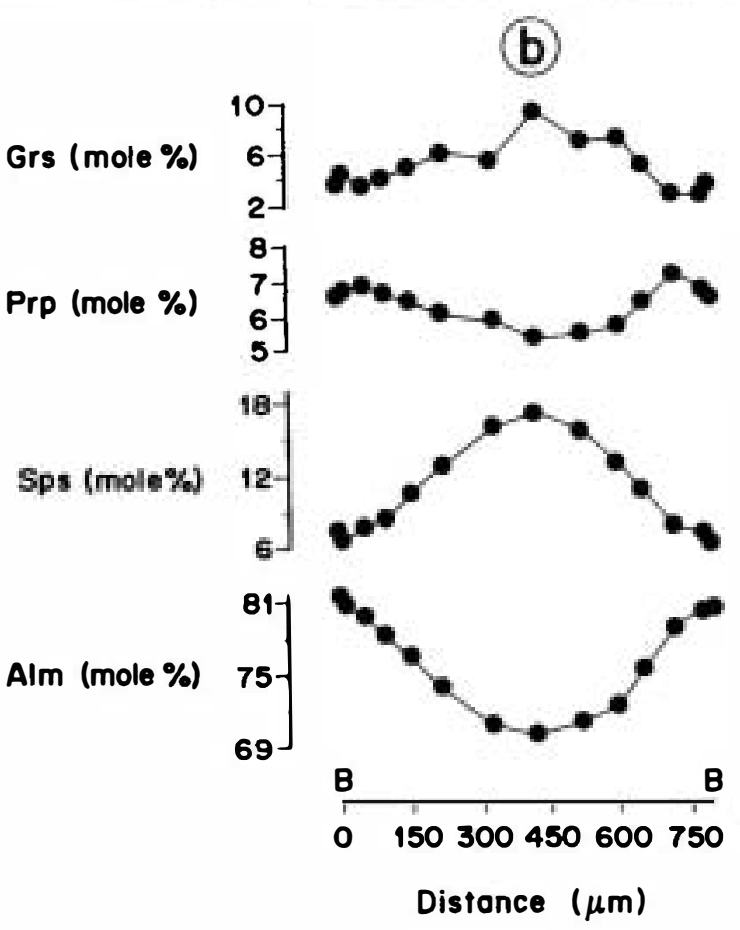

Figure 7. Profils chimiques de zonation des grenats syn-D, des métapélites et semipélites de la formation de Skhour. (a) Grenat de l'isograde Grtl +). (b) Grenats de la partie inférieure de la zone de la staurotide. B: Bords des grenats. 
du grenat sont riches en almandin, spessartine et grossulaire, et pauvres en pyrope (Table 2). Les compositions moyennes de leurs coeurs $\left(\right.$ Alm $_{61.81} \mathrm{Sps}_{21.98} \operatorname{Prp}_{4.84}$ Grs $_{11.37}$ ) et de leurs bords $\left(\right.$ Alm $_{67.22}$ Sps $_{17.10} \operatorname{Prp}_{5.69}$ Grs $\left._{9.99}\right)$ définissent un gradient de composition suggérant une croissance sous des conditions métamorphiques progrades (Tracy, 1982). Le passage de la zone du grenat à celle de la staurotide se traduit par une évolution chirnique prograde consistant en la diminution des teneurs en spessartine et grossulaire, et l'augmentation parallèle des teneurs en almandin et pyrope (Table 2).

La zonation des grenats syn- $D$, se caractérise par deux types de profils chimiques: des profils courbes, reflétant une zonation de type croissance continue (Tracy, 1982; Dempster, 1985; Ikeda, 1993; Fig. 7b), et d'autres plats, traduisant des gradients chimiques faibles à nuls (Fig. 7a). La première zonation est développée par les grenats syn-schisteux de la zone de la staurotide et de la partie inférieure de la zone du grenat, alors que la seconde est typique des grenats tardi-schisteux de l'isograde Grt + +). La tendance des profils de zonation à s' aplatir avec la diminution de la profondeur est accompagnée par la réduction de la taille des grenats, et l'évolution progressive de leurs Si vers des géométries linéaires tendant à se parallèliser avec les plans Se. L'absence d'une zonation dans les grenats de l'isograde $\mathrm{Gr}(+)$ peut être attribuée, en première instance, à leur homogénéisation pendant le pic thermique. Cette possibilité est cependant écartée puisque:

i) les grenats en question appartiennent au faciès schistes verts supérieur où les diffusions de volume sont peu effectives;

ii) les grenats de la partie supérieure de leur propre zone, présentant des tailles similaires à celles des grenats de l'isograde, ne montrent pas d'effets d'une telle homogénéisation.

Ce mode d'évolution de zonation des grenats est par contre attribué à leur cinétique de croissance variable avec la profondeur (Fig. 5): la croissance des grenats pré à syn-schisteux des zones profondes (zone de la staurotide et partie inférieure de la zone du grenat), relativement lente comme en attestent leurs $\mathrm{Si}$ sigmoïdales traduisant différents stades incrémentaux de la déformation $D_{1}$, a enregistré des variations de température importantes, et a développé, en conséquence, une zonation chimique accusée de type croissance continue (Fig. 7b). Les grenats tardi-schisteux de l'isograde englobent une Si parallèle à la Se avec laquelle elle passe en continuité sans qu'il y ait

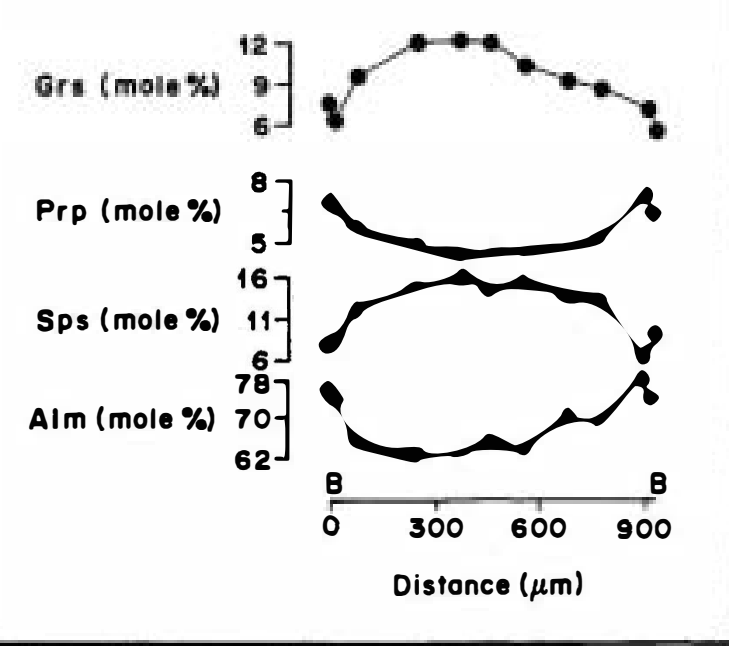

Figure 8. Profils chimiques de zonation d'un grenat syn- $D_{2 c}$ des métapélites de la partie superieure de la zone de la staurotide de la formation d'El Broumi-Ouled Hassine. B: Bords du grenat.

de variations minéralogiques des assemblages définissant ces deux fabriques planaires (Fig. 5). La croissance des ces grenats est donc relativement rapide, comme en atteste davantage leur petite taille; elle ne s'accompagne que de faibles variations thermiques qui sont à l'origine du développment d'un gradient chimique faible à nul (Fig. 7a).

Les grenats syn- $D_{2 c}$ sont riches en almandin, spessar tine et grossulaire, et pauvres en pyrope (Table 2, Fig. 8). La composition moyenne des coeurs des grenats de la formation des Skhour

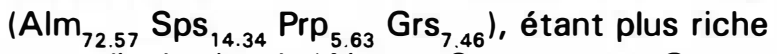
que celle des bords (Alm 79.05 Sps $_{7.01} \operatorname{Prp}_{7.56}$ Grs $_{6.38}$ ) en spessartine et grossulaire, et plus pauvre en pyrope et almandin, suggère une zonation de type croissance continue. Les mêmes gradients de composition, cette fois-ci plus accusés, sont développés par les coeurs $\left(\mathrm{Alm}_{59.80} \mathrm{Sps}_{21.72} \operatorname{Prp}_{3.25}\right.$ Grs $\left._{15.23}\right)$ et les bords $\left(\mathrm{Alm}_{74.65} \mathrm{Sps}_{9.75} \operatorname{Prp}_{5.04}\right.$ $\mathrm{Grs}_{10.56}$ ) des grenats des formations orientales. La composition chimique de ceux-ci ainsi que celle des grenats syn- $D_{1}$ du secteur central suggérent que leur croissance est de plus basse température que celle des grenats syn- $D_{2 c}$ de la formation des Skhour (Table 2). Le passage de la zone du grenat à celle de la staurotide de l'ensemble des formations est marqué par une évolution chimique prograde, révélée par une augmentation des teneurs en pyrope et almandin, et une diminution des teneurs en spessartine et grossulaire (Table 2).

Les bords externes des grenats des deux générations précitées montrent parfois une 
Table 3. Analyses chimiques représentatives des micas blancs potassiques des métapélites et semipélites de la formation de Skhour (FS) et des formations d'El Broumi-Ouled Hassine et de Lalla Tittaf (FEOL)

\begin{tabular}{|c|c|c|c|c|c|c|c|c|c|c|c|}
\hline & \multicolumn{8}{|c|}{$\mathrm{FS}$} & \multirow{2}{*}{\multicolumn{3}{|c|}{$\frac{\text { FEOL }}{\text { Syn } D_{2 c}}$}} \\
\hline & \multicolumn{4}{|c|}{ Syn $D_{1}$} & \multicolumn{4}{|c|}{ Syn $D_{2 c}$} & & & \\
\hline & $1 \mathrm{Mb} 1.1$ & $3 \mathrm{Mb} 1.1$ & $6 \mathrm{Mb} 1.1$ & $9 \mathrm{Mb} 1.1$ & $2 \mathrm{Mb} 1.1$ & $4 \mathrm{Mb} 1.1$ & $7 \mathrm{Mb} 1.1$ & $10 \mathrm{Mb} 1.1$ & $5 \mathrm{Mb} 1.1$ & $8 \mathrm{Mb} 1.1$ & $11 \mathrm{Mb} 1.1$ \\
\hline $\mathrm{SiO}_{2}$ & 48.13 & 47.54 & 46.18 & 46.93 & 47.00 & 46.80 & 46.97 & 46.38 & 47.09 & 45.77 & 47.35 \\
\hline $\mathrm{TiO}_{2}$ & 0.06 & 0.32 & 0.27 & 0.29 & 0.33 & 0.20 & 0.29 & 0.23 & 0.35 & 0.19 & 0.17 \\
\hline $\mathrm{Al}_{2} \mathrm{O}_{3}$ & 32.72 & 32.39 & 34.99 & 37.29 & 32.65 & 37.05 & 36.41 & 36.23 & 34.37 & 35.37 & 38.32 \\
\hline $\mathrm{FeO}$ & 3.42 & 3.22 & 1.15 & 0.88 & 3.38 & 1.03 & 1.50 & 2.61 & 2.33 & 1.34 & 0.66 \\
\hline MnO & 0.02 & - & 0.02 & 0.04 & - & 0.06 & 0.01 & - & 0.01 & - & 0.67 \\
\hline $\mathrm{MgO}$ & 1.42 & 1.13 & 0.59 & 0.50 & 0.95 & 0.35 & 0.34 & 0.43 & 0.88 & 0.42 & 0.34 \\
\hline $\mathrm{CaO}$ & - & 0.04 & - & - & - & 0.01 & 0.02 & - & 0.03 & - & 0.21 \\
\hline $\mathrm{Na}_{2} \mathrm{O}$ & 0.38 & 0.47 & 1.20 & 1.28 & 0.51 & 0.84 & 1.55 & 2.53 & 0.49 & 0.69 & 0.76 \\
\hline $\mathrm{K}_{2} \mathrm{O}$ & 9.69 & 10.18 & 9.42 & 8.78 & 10.40 & 8.68 & 8.80 & 7.05 & 10.17 & 10.02 & 10.08 \\
\hline Total & 95.95 & 95.3 & 93.83 & 95.99 & 95.22 & 95.02 & 95.99 & 95.46 & 96.08 & 93.8 & 98.56 \\
\hline \multicolumn{12}{|c|}{ Cations calculés sur la base de 11 oxygènes } \\
\hline$\overline{\mathrm{Si}}$ & 3.19 & 3.18 & 3.10 & 3.06 & 3.16 & 3.08 & 3.08 & 3.06 & 3.11 & 3.09 & 3.03 \\
\hline $\mathrm{Ti}$ & - & 0.02 & 0.01 & 0.01 & 0.02 & 0.01 & 0.01 & 0.01 & 0.02 & 0.01 & 0.01 \\
\hline Al(IV) & 0.81 & 0.81 & 0.90 & 0.94 & 0.84 & 0.92 & 0.92 & 0.94 & 0.89 & 0.91 & 0.97 \\
\hline Al(VI) & 1.75 & 1.74 & 1.87 & 1.93 & 1.75 & 1.95 & 1.90 & 1.87 & 1.82 & 1.90 & 1.93 \\
\hline $\mathrm{Fe}^{2+}$ & 0.19 & 0.18 & 0.06 & 0.05 & 0.19 & 0.06 & 0.08 & 0.14 & 0.13 & 0.08 & 0.04 \\
\hline$M n$ & - & - & - & - & - & - & - & - & - & - & 0.04 \\
\hline Mg & 0.14 & 0.11 & 0.06 & 0.05 & 0.10 & 0.03 & 0.03 & 0.04 & 0.09 & 0.04 & 0.03 \\
\hline $\mathrm{Ca}$ & - & - & - & - & - & - & - & - & - & - & 0.01 \\
\hline $\mathrm{Na}$ & 0.05 & 0.06 & 0.16 & 0.16 & 0.07 & 0.11 & 0.20 & 0.32 & 0.06 & 0.09 & 0.09 \\
\hline$K$ & 0.82 & 0.87 & 0.81 & 0.73 & 0.89 & 0.73 & 0.74 & 0.59 & 0.86 & 0.86 & 0.82 \\
\hline Total & 6.95 & 6.98 & 6.97 & 6.93 & 7.02 & 6.89 & 6.96 & 6.97 & 6.98 & 6.98 & 6.96 \\
\hline Paragonite & 4.69 & 5.32 & 14.36 & 16.56 & 6.13 & 11.70 & 19.25 & 32.35 & 5.64 & 8.49 & 8.97 \\
\hline Céladonite & 21.09 & 20.08 & 12.26 & 9.29 & 16.00 & 13.32 & 10.17 & 8.15 & 12.93 & 9.96 & 6.90 \\
\hline Muscovite & 74.22 & 74.60 & 73.38 & 74.53 & 77.87 & 75.07 & 70.58 & 59.50 & 80.86 & 81.12 & 82.70 \\
\hline $\mathrm{Na} / \mathrm{Na}+\mathrm{K}$ & 0.06 & 0.07 & 0.16 & 0.18 & 0.07 & 0.13 & 0.21 & 0.35 & 0.07 & 0.09 & 0.10 \\
\hline
\end{tabular}

1 et 2 : zone de la chlorite; 3,4 et $5:$ zone de la biotite; 6,7 et $8:$ zone du grenat; 9,10 et $11:$ zone de la staurotide. 
Table 4. Analyses chimiques représentatives des chlorites des métapélites et semipélites de la formation de Skhour (FS) et des formations d'El Broumi-Ouled Hassine et de Lalla Tittaf (FEOL)

\begin{tabular}{|c|c|c|c|c|c|c|c|c|c|c|}
\hline & \multicolumn{8}{|c|}{ FS } & \multirow{2}{*}{\multicolumn{2}{|c|}{$\frac{\text { FEOL }}{\text { Syn } D_{2 c}}$}} \\
\hline & \multicolumn{4}{|c|}{ Syn $D_{1}$} & \multicolumn{4}{|c|}{ Syn $D_{2 c}$} & & \\
\hline & 1Ch 1.1 & $3 \mathrm{Ch} 1.1$ & 6Ch1.1 & 6Ch2.1 & 2Ch1.1 & 4Ch1.1 & 6Ch3.1 & 7Ch1.1 & $5 \mathrm{Ch} 1.1$ & 8Ch1.1 \\
\hline$\overline{\mathrm{SiO}_{2}}$ & 23.43 & 24.14 & 24.36 & 24.17 & 24.15 & 24.65 & 24.22 & 25.68 & 23.83 & 24.53 \\
\hline $\mathrm{TiO}_{2}$ & 0.02 & 0.07 & 0.09 & 0.08 & 0.09 & 0.04 & 0.09 & 0.01 & 0.09 & 0.09 \\
\hline $\mathrm{Al}_{2} \mathrm{O}_{3}$ & 22.67 & 23.14 & 22.04 & 22.55 & 22.15 & 24.19 & 22.31 & 22.25 & 22.48 & 21.88 \\
\hline $\mathrm{FeO}$ & 33.22 & 30.57 & 26.19 & 28.17 & 30.58 & 28.17 & 25.53 & 28.05 & 31.79 & 29.45 \\
\hline $\mathrm{MnO}$ & 0.09 & 0.23 & 0.43 & 0.14 & 0.24 & 0.38 & 0.28 & 0.01 & .48 & 0.30 \\
\hline $\mathrm{MgO}$ & 9.06 & 11.06 & 13.51 & 10.62 & 11.07 & 10.74 & 14.37 & 12.90 & .40 & 10.31 \\
\hline $\mathrm{CaO}$ & - & - & 0.06 & 0.01 & - & . & & - & 0.02 & .02 \\
\hline $\mathrm{Na}_{2} \mathrm{O}$ & - & 0.03 & - & 0.05 & - & - & 0.07 & - & - & 0.02 \\
\hline $\mathrm{K}_{2} \mathrm{O}$ & - & - & 0.05 & 0.02 & - & 0.03 & - & 0.01 & 0.04 & 0.28 \\
\hline Total & 8.49 & 88.23 & 86.74 & 85.84 & 88.28 & 88.20 & 86.85 & 88.91 & 88.13 & 86.88 \\
\hline \multicolumn{11}{|c|}{ Cations calculés sur la base de 14 oxygènes } \\
\hline Si & 2.56 & 2.34 & 2.62 & 2.65 & 2.60 & 2.61 & 2.59 & 2.69 & 59 & 2.68 \\
\hline $\mathrm{Ti}$ & - & 0.01 & 0.01 & 0.01 & 0.01 & - & 0.01 & 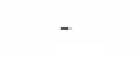 & 0.01 & 0.01 \\
\hline $\mathrm{Al}(\mathrm{IV})$ & 1.44 & 1.66 & 1.38 & 1.35 & 1.40 & 1.39 & 1.41 & 31 & 41 & 1.32 \\
\hline $\mathrm{Al}(\mathrm{VI})$ & 1.48 & 1.83 & 1.41 & 1.56 & 1.41 & 1.63 & 1.40 & 1.44 & .48 & 1.59 \\
\hline $\mathrm{Fe}^{2+}$ & 03 & 47 & 35 & 58 & 76 & 50 & 28 & 46 & 89 & .69 \\
\hline $\mathrm{Mn}$ & 0.01 & 0.02 & 0.04 & 0.01 & 0.02 & 0.03 & 0.03 & - & 0.04 & 0.03 \\
\hline $\mathrm{Mg}$ & 1.47 & 1.59 & 2 & 1.73 & 1.78 & 1.70 & 2.29 & 2.02 & 1.53 & 1.68 \\
\hline $\mathrm{Ca}$ & - & - & 0.01 & . & - & $=$ & - & = & - & - \\
\hline $\mathrm{Na}$ & . & 0.01 & - & 0.01 & - & . & 0.01 & . & . & . \\
\hline K & - & - & 0.01 & - & 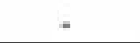 & - & - & 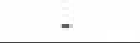 & 0.01 & 0.04 \\
\hline Total & 9.99 & 9.93 & 9.99 & 9.90 & 9.98 & 9.86 & 10.02 & 9.92 & 9.96 & 10.04 \\
\hline $\mathrm{Mg} / \mathrm{Fe}$ & 0.48 & 0.64 & 0.92 & 0.67 & 0.64 & 0.68 & 1.00 & 0.82 & 0.53 & 0.62 \\
\hline
\end{tabular}

1 et 2: zone de la chlorite; 3,4 et 5 : zone de la biotite; 6,7 et 8 : zone du grenat.

inversion de la loi de zonation normale (Figs 7 et 8) qui est attribuée, compte tenu du fait que les grenats en question sont subidiomorphes, à une rééquilibration rétrograde des bords (Tracy, 1982).

\section{Micas blancs potassiques}

Les micas blancs syn-D, de la zone de la chlorite et celle de la biotite sont des phengites $(\mathrm{Si}>3.1$; Guidotti, 1984), alors que ceux des zones du grenat et de la staurotide, moins riches en silice (Si<3.1), sont des muscovites (Table 3). Cette variation de la teneur en $\mathrm{Si}$, en fonction de l'intensité du métamorphisme, se maintient globalement dans le cas de la génération syn$D_{2 c}$ (Table 3). La diminution de la teneur en Si avec l'incrément de l'intensité du métamorphisme est accompagnée par l'augmentation de la teneur en $\mathrm{Al}$ (IV) et du rapport atomique $\mathrm{Na} / \mathrm{Na}+\mathrm{K}$ (Table 3). Cette évolution chimique prograde (Guidotti, 1984) est très accusée dans les micas syn- $D_{2 c}$ de la formation des Skhour (Table 3), qui semblent être le produit d'une évolution thermique plus intense que celle ayant conditionné I'apparition des micas syn- $D_{1}$ de la même formation ou des micas syn- $D_{2 c}$ des formations orientales.

\section{Chlorite}

Selon la classification de Hey (1954), les chlorites de l'ensemble des générations analysées (Table 4) sont des ripidolites. Sa nomenclature correspond à chamosites selon la classification de Bayliss (1975) et Bailey (1988). Le rapport atomique $\mathrm{Mg} / \mathrm{Fe}$ de chaque génération augmente de manière systématique depuis la zone de la chlorite jusqu'à celle du grenat (Table 4). Cette variation chimique prograde (Miyashiro, 1973) est relativement plus accentuée dans les chlorites syn$D_{2 c}$ de la formation des Skhour (Table 4).

\section{Biotite}

La teneur en $\mathrm{Mg}$ des biotites syn- $\mathrm{D}_{1}$ augmente depuis leur propre zone métamorphique jusqu'à la zone de la staurotide (Table 5). Cette tendance 
Table 5. Analyses chimiques représentatives des biotites des métapélites et semipélites de la formation de Skhour (FS) et des formations d'El Broumi-Ouled Hassine et de Lalla Tittaf (FEOL)

\begin{tabular}{|c|c|c|c|c|c|c|c|c|c|c|}
\hline & \multicolumn{6}{|c|}{ FS } & \multirow{2}{*}{\multicolumn{4}{|c|}{$\frac{\text { FEOL }}{\text { Syn } D_{2 c}}$}} \\
\hline & \multicolumn{3}{|c|}{ Syn $D_{1}$} & \multicolumn{3}{|c|}{ Syn $D_{2 c}$} & & & & \\
\hline & 3Bio1.1 & 6Bio1.1 & 9Bio1.1 & 4Bio1.1 & 7Bio1.1 & 10Bio 1.1 & 5Bio1.1 & 8Bio1.1 & 11Bio1.1 & $11 \mathrm{Bio} 2.1$ \\
\hline$\overline{\mathrm{SiO}_{2}}$ & 37.57 & 37.15 & 34.88 & 38.90 & 36.55 & 34.99 & 38.89 & 36.58 & 34.92 & 35.03 \\
\hline $\mathrm{TiO}_{2}$ & 2.02 & 1.43 & 1.86 & 1.70 & 1.60 & 1.91 & 1.68 & 1.55 & 1.90 & 1.85 \\
\hline $\mathrm{Al}_{2} \mathrm{O}_{3}$ & 18.49 & 16.85 & 19.62 & 18.89 & 18.60 & 19.17 & 18.91 & 18.65 & 19.16 & 19.22 \\
\hline $\mathrm{FeO}$ & 22.68 & 22.65 & 21.11 & 19.50 & 21.50 & 20.50 & 19.60 & 21.60 & 20.48 & 21.01 \\
\hline $\mathrm{MnO}$ & 0.09 & 0.06 & 0.30 & 0.29 & 0.35 & 0.29 & 0.26 & 0.36 & 0.35 & 0.23 \\
\hline $\mathrm{MgO}$ & 6.92 & 8.38 & 8.34 & 7.24 & 8.20 & 8.70 & 7.34 & 8.30 & 8.67 & 8.70 \\
\hline $\mathrm{CaO}$ & 0.09 & 0.06 & - & 0.14 & 0.16 & - & 0.16 & 0.14 & - & - \\
\hline $\mathrm{Na}_{2} \mathrm{O}$ & 0.08 & 0.01 & 0.11 & 0.08 & 0.09 & 0.04 & 0.06 & 0.10 & 0.06 & 0.11 \\
\hline $\mathrm{K}_{2} \mathrm{O}$ & 9.06 & 8.68 & 9.66 & 9.01 & 9.79 & 9.99 & 9.00 & 9.76 & 10.00 & 10.02 \\
\hline Total & 96.99 & 95.27 & 95.88 & 95.75 & 96.84 & 95.59 & 95.90 & 97.04 & 95.55 & 96.18 \\
\hline \multicolumn{11}{|c|}{ Cations calculés sur la base de 11 oxygènes } \\
\hline $\mathrm{Si}$ & 2.82 & 2.85 & 2.67 & 2.91 & 2.77 & 2.68 & 2.90 & 2.76 & 2.68 & 2.67 \\
\hline $\mathrm{Ti}$ & 0.11 & 0.08 & 0.11 & 0.10 & 0.09 & 0.11 & 0.09 & 0.09 & 0.11 & 0.11 \\
\hline$A \mid(I V)$ & 1.18 & 1.15 & 1.33 & 1.09 & 1.23 & 1.32 & 1.10 & 1.24 & 1.33 & 1.33 \\
\hline Al(VI) & 0.45 & 0.37 & 0.44 & 0.58 & 0.43 & 0.41 & 0.57 & 0.42 & 0.39 & 0.39 \\
\hline $\mathrm{Fe}^{2+}$ & 1.42 & 1.45 & 1.35 & 1.22 & 1.36 & 1.31 & 1.22 & 1.36 & 1.31 & 1.34 \\
\hline $\mathrm{Mn}$ & 0.01 & - & 0.02 & 0.02 & 0.02 & 0.02 & 0.02 & 0.02 & 0.02 & 0.01 \\
\hline $\mathrm{Mg}$ & 0.77 & 0.96 & 0.95 & 0.81 & 0.92 & 0.99 & 0.82 & 0.93 & 0.99 & 0.99 \\
\hline $\mathrm{Ca}$ & 0.01 & - & - & 0.01 & 0.01 & - & 0.01 & 0.01 & - & - \\
\hline $\mathrm{Na}$ & 0.01 & - & 0.02 & 0.01 & 0.01 & 0.01 & 0.01 & 0.01 & 0.01 & 0.02 \\
\hline$\underline{K}$ & 0.87 & 0.85 & 0.94 & 0.86 & 0.94 & 0.98 & 0.86 & 0.94 & 0.98 & 0.97 \\
\hline Total & 7.65 & 7.71 & 7.83 & 7.61 & 7.78 & 7.83 & 7.60 & 7.78 & 7.83 & 7.84 \\
\hline $\mathrm{Mg} / \mathrm{Fe}$ & 0.54 & 0.66 & 0.70 & 0.66 & 0.68 & 0.76 & 0.67 & 0.68 & 0.76 & 0.74 \\
\hline
\end{tabular}

3, 4 et 5: zone de la biotite; 6, 7 et 8: zone du grenat; 9, 10 et 11: zone de la staurotide.

des biotites à s'enrichir en $\mathrm{Mg}$ avec I'incrément de la température (Guidotti, 1984) caractérise également la génération syn- $D_{2 c}$ (Table 5$)$. Celleci montre des teneurs en $\mathrm{Mg}$ nettement supérieures à celles des biotites syn- $D_{1}$ de la formation des Skhour (Table 5).

\section{Plagioclases}

La variation de la teneur en anorthite des plagioclases syn- $D_{1}$ évolue parallèlement à l'augmentation de la température: le plagioclase de la zone de la biotite est une albite $\left(A n_{0.87-1.55}\right)$, alors que ceux des zones métamorphiques plus profondes sont des oligoclases relativement plus riches en $\mathrm{CaO}$ dans la zone de la staurotide $\left(A n_{19}\right)$ que dans celle du grenat $\left(A n_{12}\right)$. Les plagioclases syn- $D_{2 c}$ analysés appartiennent aux zones du grenat et de la staurotide. II s'agit d'oligoclases $\left(A n_{14.15}\right)$, ne montrant pas de variations significatives en anorthite.

\section{Oxydes de Fe-Ti}

Dans le système ternaire $\mathrm{FeO}-\mathrm{Fe}_{2} \mathrm{O}_{3}-\mathrm{TiO}_{2}$, le contenu en $\mathrm{TiO}_{2}$ des oxydes syn- $\mathrm{D}_{1}$ diminue avec l'augmentation de la température, parallèlement à un accroissement des teneurs en ilménite: la solution solide de cette génération, représentée par la proportion de molécule de rutile dans les oxydes $\mathrm{Fe}-\mathrm{Ti}$ de la zone de la biotite $\mathrm{Rt}_{84,48}$ $\left.\|_{15.52}\right)$, évolue vers des compositions proches du terme final ilménite dans la zone du grenat $\left(R t_{7.11}, \| m_{92.89}\right)$ et celle de la staurotide $\left(R^{5.48}\right.$ $\left.\mathrm{II}_{93.02}\right)$. Cette transformation régionale du rutile en ilménite avec l'incrément de la température caractérise également la génération syn- $D_{2 c}$. Celle-ci est représentée par le terme final rutile dans la zone de la biotite $\left(\mathrm{Rt}_{92.05} \mathrm{\| m} \mathrm{m}_{7.95}\right)$, présente une composition intermédiaire dans la zone du grenat $\left(R t_{41.83} \mid l m_{58.17}\right)$ et tend vers une composition proche de celle de l'ilménite s.s. dans la zone de la staurotide $\left(\mathrm{Rt}_{9.22} \mathrm{llm}_{90.78}\right)$. L'enrichissement en rutile de cette deuxième génération par rapport à la première est rapporté à l'augmentation de la pression (Frost, 1991) au cours des chevauchements (cas de la formation des Skhour) et du plissement (cas du bloc dévono-viséen oriental) de deuxième phase tectonique. 


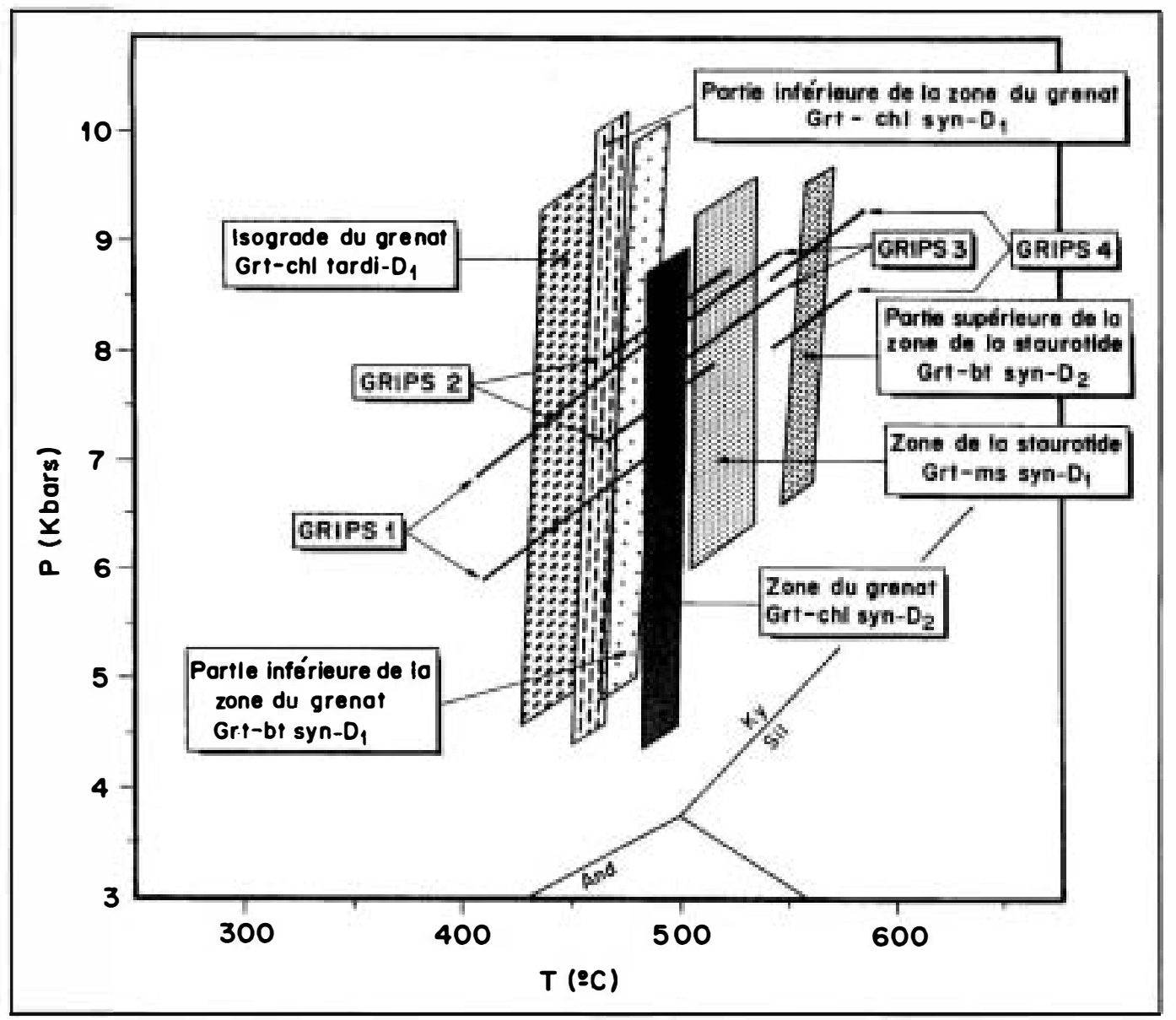

Figure 9. Représentation graphique des résultats thermobarométriques obtenus sur des assemblages métamorphiques syn-D, et syn- $D_{2 c}$ de la zone du grenat et de celle de la staurotide de la formation de Skhour. Thermomètres: grt-chl (Ghent et al., 1987); grt-bio (Ferry et Spear, 1978; Hodges et Spear, 1982; Hoinkes, 1986); grt-ms (Hynes et Forest, 1988). Baromètre: GRIPS (Bohlen et Liotta, 1986), appliqué, $d^{\prime} u n e$ part, aux assemblages syn- $D_{1}(1)$ et syn- $D_{2 c}(2)$ de la zone du grenat et, d'autre part, aux assemblages syn-D, (3) et syn- $D_{2 c}$ (4) de la zone de la staurotide. Le point triple des silicates d'alumine est celui de Holdaway (1971). Les abréviations des minéraux sont conformes à celles proposées par Kretz (1983).

\section{GEOTHERMOBAROMETRIE}

L'étude thermobarométrique a pour objet de quantifier les conditions $P-T$ maximales et minimales du pic thermique. Ces conditions seront calculées sur des assemblages minéralogiques syn- $D_{2 c}$ des formations $d^{\prime} E I$ Broumi-Ouled Hassine et de Lalla Tittaf, en position structurale supérieure avant la mise en jeu de la tectonique extensive qui a provoqué par la suite leur mise en place au sommet de l'édifice orogénique, et dans la formation des Skhour du secteur central sous jacent. Les conditions $P$ - $T$ du plissement $P$, seront également quantifiées dans cette dernière formation. Elles n'ont pas pu être calculées dans les formations orientales où les plans de schistosité $S_{1}$, qui n'y sont que localement rencontrés, ne sont définis que par des assemblages à chlorite d'intérêt thermobarométrique limité. Les conditions maximales du pic thermique dans le secteur central sont, en fait, atteintes par la ZCKM. Elles $n^{\prime} y$ ont pas pu être évaluées en raison de la nature de ses lithologies essentiellement semipélitiques (métaconglomérats et métagrauwackes) à assemblages ne présentant généralement pas d'application thermobarométrique. Ces difficultés ont été accentuées par le caractère rétrograde des associations minérales définissant la foliation régionale individualisée au cours de la réactivation ultérieure de la ZCKM en faille normale.

Des analyses des minéraux à l'équilibre textural ont été choisies pour quantifier ces conditions de pression et de température. Dans la zone du grenat et celle de la staurotide, la composition des grenats utilisée en thermobarométrie correspond à celle des bords entrant en équilibre textural direct avec les autres phases de la 


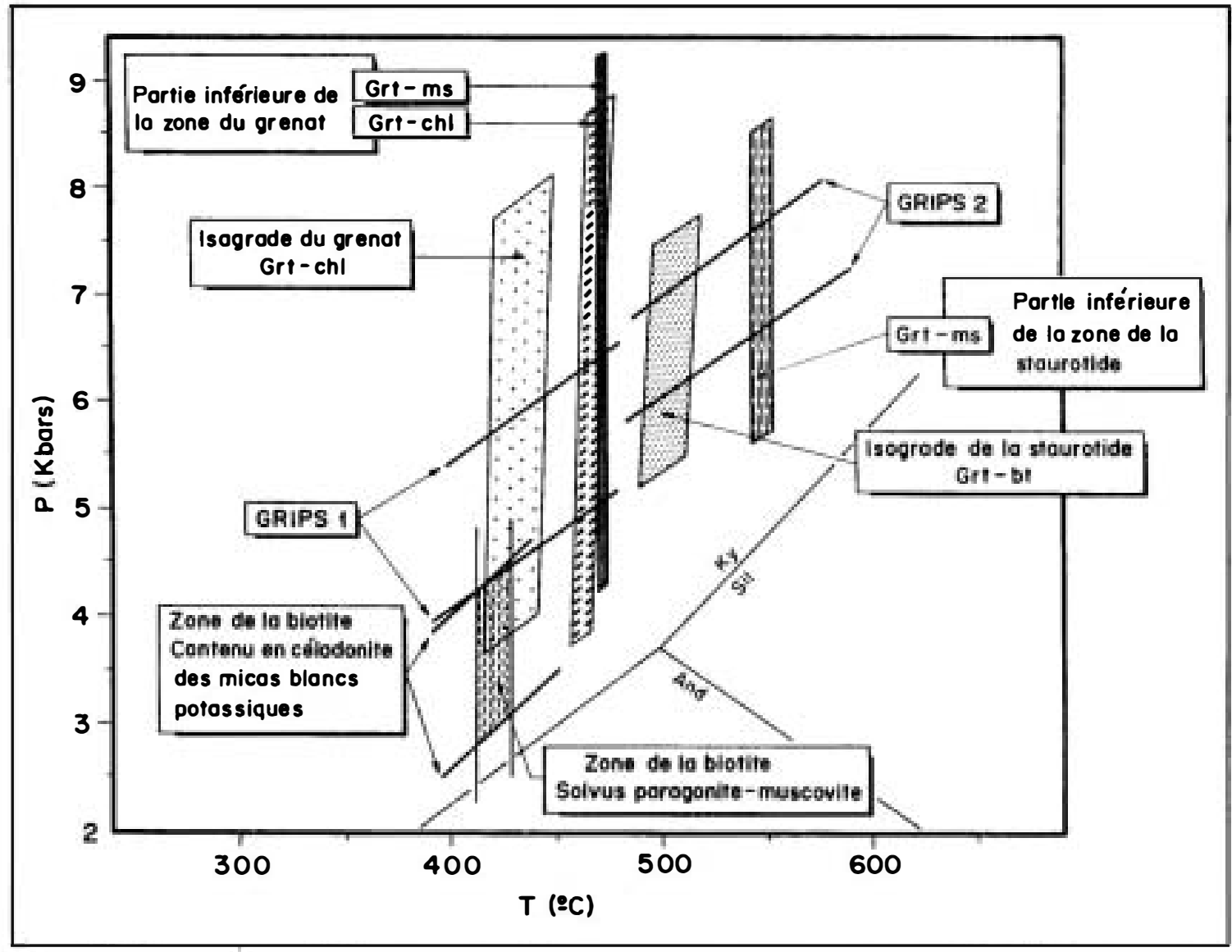

Figure 10. Représentation graphique des résultats thermobarométriques obtenus sur les assemblages syn- $D_{2 c}$ des zones de la biotite, du grenat et de la staurotide des formations d'El Broumi-Ouled Hassine et de Lalla Tittaf. Les calculs de la température et de la pression dans la zone de la biotite ont été respectivement réalisés par la thermométrie de solvus paragonite-muscovite (Eugster et al., 1972) et par le contenu en céladonite des micas blancs potassiques (Massone et Schreyer, 1987). Le baromètre GRIPS a été appliqué dans la zone du grenat (1) et celle de la staurotide (2). Les autres abréviations sont identiques à celles de la Figure 6.

matrice, et ne montrant généralement pas d'inversions de la zonation normale.

Les tentatives d'estimation des conditions $P$ - $T$ des assemblages syn- $D_{1}$ et syn- $D_{2 c}$ de la zone de la chlorite de la formation des Skhour, par utilisation conjointe de la thermométrie de solvus paragonite-muscovite (Eugster et al., 1972) et de la barométrie basée sur le contenu en céladonite des micas blancs potassiques (Massone et Schreyer, 1987), ont donné des résultats trop faibles pour être pris en considération.

Dans la zone de la biotite, les seuls résultats significatifs ont été obtenus par l'assemblage syn- $D_{2 c}$ de la formation d'El Broumi-Ouled Hassine (Fig. 10). La thermométrie de solvus paragonite-muscovite (Eugster et al., 1972) a permis de calculer la température minimale. Quant à la pression, elle a été estimée en utilisant les teneurs en céladonite des micas blancs potassiques (Massone et Schreyer, 1987). Les pressions calculées, compte tenu du fait que le feldspath potassique fait défaut dans les métapélites et semipélites des Rehamna, correspondent à des valeurs minimales.

Dans la zone du grenat, le calcul de la température a été basé sur différentes réactions d'échange entre les phases des assemblages syn$D_{1}$ et syn- $D_{2 c}$ (Figs 9 et 10). Les résultats significatifs ont été obtenus par les réactions d'échange Fe-Mg entre biotite et grenat (Ferry et Spear, 1978; Hodges et Spear, 1982; Hoinkes, 1986), chlorite et grenat (Ghent et al., 1987) et muscovite et grenat (Hynes et Forest, 1988). Le calibrage utilisé dans ce dernier thermomètre correspond à l'équation 9 de Hynes et Forest (1988), en raison de sa dépendance pratiquement nulle de la pression. Le modèle d'activité utilisé dans le cas du grenat est celui de Ganguly et Saxena (1984), avec des corrections des effets du calcium basées sur l'expression de Hoinkes (1986). Les grenats de la partie supérieure de leur propre zone 
Table 6. Résultats thermobarométriques obtenus dans la formation des Skhour (Rehamna centraux) et dans les formations d'El Broumi-Ouled Hassine et de Lalla Tittaf (Rehamna orientaux)

\begin{tabular}{|c|c|c|c|c|}
\hline Formations & Déformation & $\begin{array}{l}\text { Zones } \\
\text { métamorphiques }\end{array}$ & $\mathrm{T}\left({ }^{\circ} \mathrm{C}\right)$ & $P$ (kbar) \\
\hline \multirow[t]{4}{*}{ Skhour } & $D_{1}$ & Grenat & $460 \pm 30$ & 7 \\
\hline & & Staurotide & $520 \pm 15$ & $8 \pm 0.5$ \\
\hline & Stades pré à syn $D_{2 c}$ & Grenat & $495 \pm 10$ & $8 \pm 0.5$ \\
\hline & & Staurotide & $560 \pm 10$ & $9 \pm 0.5$ \\
\hline El Broumi-Ouled Hassine & Stades pré à syn $D_{2 c}$ & Biotite & $420 \pm 10$ & $4 \pm 1$ \\
\hline et Lalla Tittaf & & $\begin{array}{l}\text { Grenat } \\
\text { Staurotide }\end{array}$ & $\begin{array}{l}448 \pm 10 \\
532 \pm 10 \\
\end{array}$ & $\begin{array}{l}5 \pm 0.5 \\
7\end{array}$ \\
\hline
\end{tabular}

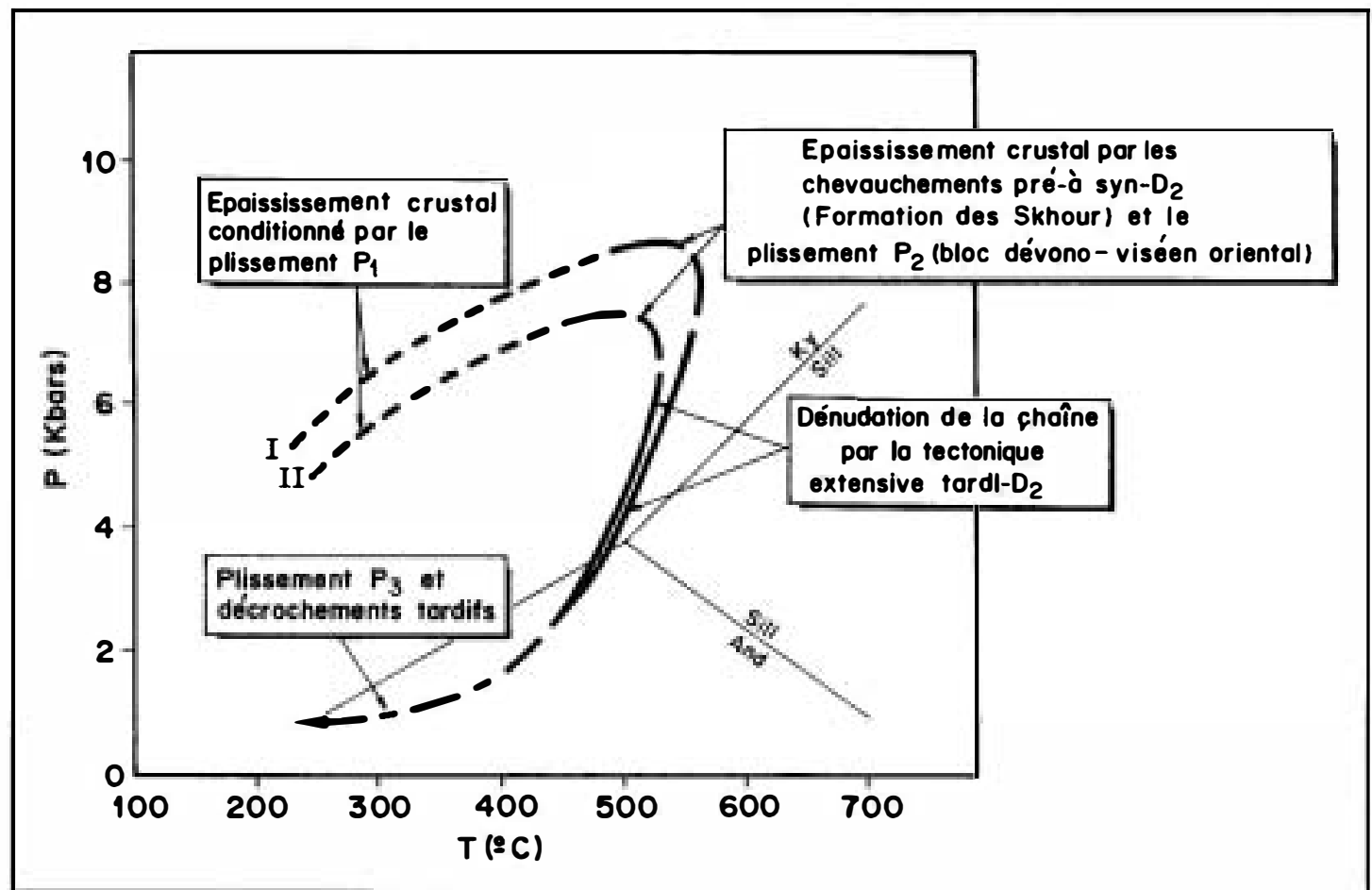

Figure 11. Chemins $\mathrm{P}-\mathrm{T}$ empruntés par les roches de la zone de la staurotide de la formation de Skhour (I) et des formations d'El Broumi-Ouled Hassine et de Lalla Titta (III). Dans les Rehamna orientaux, la partie syn-D, du trajet P-T n'est que supposée. Le point triple des silicates d'alumine est celui de Holdaway (1971).

métamorphique présentent souvent des teneurs en spessartine élevées. De ce fait, les résultats privilégiés sont ceux obtenus par les calibrages grenat-biotite (Hoinkes, 1986), grenat-chlorite (Ghent et al., 1987) et grenat-muscovite (Hynes et Forest 1988), qui prennent en compte l'activité de $\mathrm{Mn}$ dans le grenat. Quant à la pression, elle a été évaluée par le baromètre grenat-rutile-ilménite-plagioclase-quartz ('GRIPS'; Bohlen et Liotta, 1986).

Dans la zone de la staurotide, la température a été estimée par les thermomètres grenat-biotite (Ferry et Spear, 1978; Hodges et Spear, 1982) et grenat-muscovite (Hynes et Forest, 1988; Figs 9 et 10). Le calibrage utilisé dans ce dernier thermomètre correspond toujours à l'équation 9. Ici encore, le calcul de la pression a été toujours réalisé par le baromètre GRIPS (Bohlen et Liotta, 1986; Figs 9 et 10).

Les resultats thermobarométriques obtenus dans l'ensemble des zones métamorphiques précitées sont reportés dans la Table 6 et dans les Figures 9 et 10. Il est à remarquer que dans la zone de la staurotide de la formation des Skhour, les assemblages syn- $D_{1}$ abondent régionalement. Les conditions $P-T$ qui y sont 
calculées ont couvert toute la zone. Les assemblages syn- $D_{2 c}$ sont, par contre, restreints à la bande de déformation syn-schisteuse localisée au contact de la faille normale de la $\mathrm{ZCOZ}$, où ils sont presque complètement oblitérés par les associations minérales syn- $D_{2 e}$ : les quelques reliques encore préservés appartiennent à la partie supérieure de la zone. Les conditions $P$ - $T$ calculées sur ses reliques, correspondant donc aux valeurs minimales du pic thermique, expliquent bien les différences, apparement peu significatives, entre les conditions $P$ - $T$ du plissement $P_{1}$ et celles de la déformation $D_{2 c}$.

\section{EVOLUTION TECTONOTHERMALE ET CONCLUSIONS}

L'integration des caractéristiques métamorphiques et structurales fait ressortir deux épisodes tectonothermaux (Fig. 11): un épisode $M_{1}$ prograde, d'affinité barrovienne, régi par un régime tectonique compressif (plissement $P_{1}$ et chevauchements syn- $D_{2 c}$ ), et un autre, $M_{2}$, rétrograde, contrôlé par la tectonique extensive et par le plissement et les décrochements $D_{3}$.

L'épisode $M_{1}$ est une phase d'épaississement crustal qui s'est initiée par la phase de plis $P_{\text {, }}$ sous un climat métamorphique de moyenne $P / T$. Les conditions $P$ - $T$ maximales ( $c$ 'est à dire celles de la zone à staurotide) de ce plissement dans la formation des Skhour sont de $520^{\circ} \mathrm{C}$ à $8 \mathrm{kbar}$. En considérant une densité normale des matériaux crustaux ( $\left.3.5 \mathrm{~km} \mathrm{kbar}^{-1}\right)$, ces conditions suggèrent un gradient thermique faible, de $18^{\circ} \mathrm{C} \mathrm{km}^{-1}$, qui situe l'évolution métamorphique du côté de la limite des pressions élevées du régime barique de moyenne $P / T$.

L'épaississement crustal culmine ensuite par charriage des formations cambro-ordoviciennes (formations des Skhour et de Jorf El Ahmar) vers I'O à NO. L'évolution métamorphique associée à ces chevauchements dans la nappe des Skhour est un réchauffement compressif qui a aboutit à I'établissement des conditions $P$ - $T$ maximales du paroxysme thermique (Fig. 11). Celles-ci sont de $495^{\circ} \mathrm{C}$ à $8 \mathrm{kbar}$ dans la zone du grenat, et $560^{\circ} \mathrm{C}$ à $9 \mathrm{kbar}$ dans la zone de la staurotide (Fig. 9). Les conditions minimales sont par contre établies dans les formations d'El Broumi-Ouled Hassine et de Lalla Tittaf; elles sont de $420^{\circ} \mathrm{C}$ à 4 kbar dans la zone de la biotite, $448^{\circ} \mathrm{C}$ à 5 kbar dans la zone du grenat, et $532^{\circ} \mathrm{C}$ à $7 \mathrm{kbar}$ dans la zone de la staurotide (Fig. 10). Le pic thermique dans ces formations orientales est régi par le plissement $P_{2}$ qui est à l'origine de leur structuration régionale. L'évolution métamorphique associée à ce plissement est également un réchauffement compressif (Fig. 11), comme en atteste la superposition d'assemblages minéralogiques à grenat du faciès amphibolite supérieur, définissant régionalement la schistosité $\mathrm{S}_{2 \mathrm{c}^{\prime}}$ aux assemblages épizonaux à chlorite marquant les plans de schistosité $S_{1}$ épargnés des processus de transposition dans les charnières $P_{2}$.

Les conditions $P-T$ du pic thermique régional sont atteintes sous un gradient de $25^{\circ} \mathrm{C} \mathrm{km}^{-1}$ dans le bloc dévono-viséen oriental, alors que dans la formation des Skhour elles sont toujours établies sous un gradient thermique de $18^{\circ} \mathrm{C}$ $\mathrm{km}^{-1}$. Ces différences de gradient relèvent des différences d'enfouissement des deux secteurs, et donc de leurs positions structurales différentes dans l'édifice orogénique avant la mise en jeu de la tectonique extensive.

En principe, les perturbations thermiques engendrées par un régime tectonique compressif évoluent par la suite vers une structure thermique stable. L'acquisition d'une telle structure se réalise par relaxation thermique induisant un réchauffement décompressif (England et Thompson, 1984) qui conditionnerait, dans le cas du massif des Rehamna, l'établissement du pic thermique régional dans le champ de stabilité de la sillimanite. Cette relaxation thermique a été cependant bloquée par la tectonique extensive, comme en atteste l'absence d'assemblages de ce silicate d'alumine à la surface d'érosion actuelle au niveau et hors du secteur étudié. Le pic thermique devrait être, en conséquence, proche du pic de pression. La tectonique extensive a assuré la mise en place du bloc dévono-viséen au sommet de l'édifice orogénique, et a achevé l'accordance, déjà initiée au cours des chevauchements syn- $D_{2 c^{\prime}}$ des autres secteurs du massif. Elle est accompagnée d'une évolution métamorphique rétrograde fortement décompressive (Fig. 11): la forte condensation des zones métamorphiques (Fig. 3), l'évolution fréquente de la $S_{2 e}$ des détachements vers une foliation mylonitique par réduction de la taille des grains des roches, et le développement d'assemblages à disthène, puis à andalousite, rendent compte d'une telle évolution métamorphique. Néamoins, cet événement métamorphique devrait initialement $s^{\prime}$ opérér selon un trajet $P$ - $T$ à pente assez forte (variations thermiques faibles selon un trajet isotherme), pour que certains minéraux (grenat et disthène) similaires à ceux de la déformation $D_{2 c}$ puissent encore se développer avant que le chemin rétrograde franchisse le champ de stabilité de l'andalousite (Fig. 11). 
En fin d'évolution tectonothermale, le trajet rétromorphique est associé au plissement et aux décrochements de troisième phase tectonique (Fig. 11); il s'opère à basse pression depuis les conditions du faciès amphibolite (croissance post $-\mathrm{D}_{2}$ de la staurotide) jusqu'à celles du faciès schistes verts (rétromorphose du grenat, disthène ou staurotide en biotite, chlorite et/ou muscovite).

\section{REMERCIEMENTS}

Nous tenons à remercier vivement I"Instituto Español de Cooperación con El Mundo Arabe' qui a accordé une bourse de recherches pour le premier auteur.

\section{Editorial Handling - L. Tack}

\section{REFERENCES}

Aghzer, A. M. 1994. Evolution tectonothermale du massif hercynien des Rehamna (Zone mésétienne centrale, Maroc). Thèse d'Etat. 358p. Université Complutense, Madrid, Spain.

Aghzer, A. M. et Arenas, R. 1995. Détachements et tectonique extensive dans le massif hercynien des Rehamna (Maroc). Journal African Earth Sciences 21, 383-393.

Bailey, S. W. 1988. Chlorites: structures and crystal chemistry. Reviews Mineralogy 19, 347-403.

Bayliss, P. 1975. Nomenclature of the trioctahedral chlorites. Canadian Mineralogist 13, 178-180.

Bohlen, S. R. and Liotta, J. L. 1986. A barometer for garnet amphibolites and garnet granulites. Journal Petrology 27, 1025-1034.

Corsini, M. 1988. Lithostratigraphie et tectonique des terrains paléozoïques dans les Rehamna et la Méséta côtière. (Méséta marocaine hercynienne). Thèse. 150p. Université Aix-Marseille, France.

Corsini, M., Cornée, J. J., Müller, J. et Vauchez, A. 1988. Cisaillement synmétamorphe et déplacement tangentie vers le SW dans les Rehamna (Maroc hercynien). Comptes Rendus Académie Sciences Paris 306, 1389-1394.

Dempster, T. J. 1985. Garnet zoning and metamorphism of the Barrovian Type Area, Scotland. Contributions Mineralogy Petrology 89, 30-38.

England, P. C. and Thompson, A. B. 1984. Pressuretemperature-time paths of regional metamorphism. I. Heat transfer during the evolution of regions of thikened continental crust. Journal Petrology 25, 894-928.

Eugster, H. P., Albee, A. L., Bence, A. E., Thompson, J. B. and Waldbaum, D. R. 1972. The two-phase region and excess mixing properties of paragonite-muscovite crystalline solutions. Journal Petrology 13, 147-179

Ferry, J. M. and Spear, F. S. 1978. Experimental calibration of the partitioning of $\mathrm{Fe}$ and $\mathrm{Mg}$ between biotite and garnet. Contributions Mineralogy Petrology 66, 113-117.

Frost, B. R. 1991. Stability of oxide minerals in metamorphic rocks. In: Oxide Minerals. Reviews in Mineralogy (Edited by Lindsley, D. H.) Mineralogical Society America 25, 469487.

Ganguly, J. and Saxena, S. K. 1984. Mixing properties of aluminosilicate garnets: constraints from natural and experimental data, and applications to geothermobarometry. American Mineralogist 69, 88-97.

Ghent, E. D., Stout, M. Z., Black, P. M. and Brothers, R. N. 1987. Chloritoid-bearing rocks associated with blueschists and eclogites, northern New Caledonia. Journal Metamorphic Geology 5, 239-254.

Guidotti, C. V. 1984. Micas in metamorphic rocks. In: Micas. Reviews in Mineralogy (Edited by Bailey, S. W.) Mineralogical Society America, Special Publication 13, 357-467.

Hey, M. H. 1954. A new review of the chlorites. Mineralogical Magazine 30, 277-292.

Hodges, K. V. and Spear, F. S. 1982. Geothermobarometry and the $\mathrm{Al}_{2} \mathrm{SiO}_{5}$ triple point at Mt Moosilanke, New Hampshire. American Mineralogist 67, 1118-1134.

Hoeppfner, C., Jenny, P. et Piqué, A. 1975. La tectogenèse hercynienne dans le massif des Rehamna (Maroc). Ecaillages et coulisements synmétamorphiques en bordure du môle côtier mésétien. Sciences Géologiques Bulletin Strasbourg 3, 179-203.

Hoinkes, G. 1986. Effect of grossular-content in garnet in the partitioning of $\mathrm{Fe}$ and $\mathrm{Mg}$ between garnet and biotite. An empirical investigation on staurolite zone samples from the Austroalpine Schneeberg Complex. Contributions Mineralogy Petrology 92, 393-399.

Holdaway, M. J. 1971. Stability of andalousite and the aluminium silicate phase diagrams. American Journal Science 271, 97-131.

Hynes, A. and Forest, R. C. 1988. Empirical garnet-muscovite geothermometry in low-grade metapelites, Selwyn Range (Canadian Rockies). Journal Metamorphic Geology 6, 297 309.

Ikeda, T. 1993. Compositional zoning patterns of garnet during prograde metamorphism from the Yanai district, Ryoke metamorphic belt, southwest Japan. Lithos 30 , 109-121.

Jenny, P. 1974. Contribution à la géologie structurale des Rehamna. Le matériel paléozoïque et son évolution hercynienne dans le centre du massif. Thèse $3^{\mathrm{eme}} \mathrm{cycle}$ 120p. Université Loui-Pasteur, Strasbourg, France

Kretz, R. 1983. Symbols for rock-forming minerals. American Mineralogist 68, 277-279.

Lagarde, J. L. and Michard, A. 1986. Stretching normal to the regional thrust displacement in a thrust-wrench shear zone. Rehamna massif, Morocco. Journal Structural Geology 8, 483-492.

Massone, H. J. et Schreyer, W. 1987. Phengite geobarometry based on the limiting assemblage with $\mathrm{K}$-feldspar, phlogopite and quartz. Contributions Mineralogy Petrology 96, 212-224.

Michard, A. 1968. La zone à staurotide et disthène des Rehamna (Maroc hercynien): une échine thermique tardi tectonique. Comptes Rendus Académie Sciences Paris 266, 1813-1816.

Miyashiro, A. 1973. Metamorphism and metamorphic belts. 492p. George Alan \& Unwin Ltd, London.

Piqué, A. 1972. Contribution à la géologie structurale des Rehamna (Méséta marocaine méridionale). Le matériel paléozoïque et son évolution hercynienne dans l'Ouest du massif. These $3^{\text {eme }}$ cycle $101 \mathrm{p}$. Université Louis-Pasteur, Strasbourg, France.

Sougy, J. 1976. Existence d'une importante tectonique tangentielle avec nappes repliées dans les Jbilet occidentales et centrales (méséta marocaine hercynienne). Travail Laboratoire Sciences Terre, St-Jérôme, Marseille, France, série B12, 41p.

Sougy, J., Muller, J. et Poutchkovsky, A. 1978. Tectonique de nappes dans la méséta marocaine hercynienne, Jbilet Rehamna. $6^{\text {eme }}$ Réunion Annals Science Terre, Orsay, France, 371p.

Tracy, R. J. 1982. Compositional zoning and inclusions in metamorphic minerals. In: Characterization of Metamorphism through Mineral Equilibria. Reviews in Mineralogy (Edited by Ferry, J. M.) Mineralogical Society America 10, 355-397. 